\title{
Geochronology and thermochronology of Cretaceous plutons and metamorphic country rocks, Anyui-Chukotka fold belt, North East Arctic Russia
}

\author{
E. L. Miller ${ }^{1}$, S. M. Katkov ${ }^{2}$, A. Strickland ${ }^{1}$, J. Toro ${ }^{3}$, V. V. Akinin ${ }^{4}$, and T. A. Dumitru ${ }^{1}$ \\ ${ }^{1}$ Department of Geological and Environmental Sciences, Stanford University, Stanford, CA, USA \\ ${ }^{2}$ The Geological Institute, Russian Academy of Science, Moscow, Russia \\ ${ }^{3}$ Department of Geology \& Geography, West Virginia University, Morgantown, WV, USA \\ ${ }^{4}$ North-East Interdisciplinary Science Research Institute FEB RAS, Magadan, Russia
}

\begin{abstract}
U-Pb isotopic dating of seven granitoid plutons and associated intrusions from the Bilibino region (Arctic Chukotka, Russia) was carried out using the SHRIMP-RG. The crystallization ages of these granitoids, which range from approximately $116.9 \pm 2.5$ to $108.5 \pm 2.7 \mathrm{Ma}$, bracket two regionally significant deformational events. The plutons cut folds, steep foliations and thrust-related structures related to sub-horizontal shortening at lower greenschist facies conditions $\left(D_{1}\right)$, believed to be the result of the collision of the Arctic Alaska-Chukotka microplate with Eurasia along the South Anyui Zone (SAZ). Deformation began in the Late Jurassic, based on fossil ages of syn-orogenic clastic strata, and involves strata as young as early Cretaceous (Valanginian) north of Bilibino and as young as HauterivianBarremian, in the SAZ. The second phase of deformation $\left(D_{2}\right)$ is developed across a broad region around and to the east of the Lupveem batholith of the Alarmaut massif and is interpreted to be coeval with magmatism. $D_{2}$ formed gently-dipping, high-strain foliations $\left(S_{2}\right)$. Growth of biotite, muscovite and actinolite define $S_{2}$ adjacent to the batholith, while chlorite and white mica define $S_{2}$ away from the batholith. Sillimanite ( \pm andalusite) at the southeastern edge the Lupveem batholith represent the highest grade metamorphic minerals associated with $D_{2} . D_{2}$ is interpreted to have developed during regional extension and crustal thinning. Extension directions as measured by stretching lineations, quartz veins, boudinaged quartz veins is NE-SW to NW-SE. Mapped dikes associated with the plutons trend mostly NW$\mathrm{SE}$ and indicate NE-SW directed extension. ${ }^{40} \mathrm{Ar} /{ }^{39} \mathrm{Ar}$ ages from $S_{2}$ micas range from $109.3 \pm 1.2$ to $103.0 \pm 1.8 \mathrm{Ma}$ and are interpreted as post-crystallization cooling ages following a protracted period of magmatism and high heat flow.
\end{abstract}

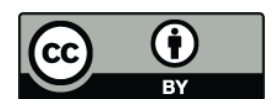

Correspondence to: E. L. Miller (elmiller@stanford.edu)
Regional uplift and erosion of many kilometers of cover produced a subdued erosional surface prior to the eruption of volcanic rocks of the Okhotsk-Chukotka volcanic belt (OCVB) whose basal units ( $\sim 87 \mathrm{Ma})$ overlie this profound regional unconformity. A single fission track age on apatite from granite in the Alarmaut massif yielded an age of $90 \pm 11 \mathrm{Ma}$, in good agreement with this inference.

\section{Introduction}

The Anyui-Chukotka foldbelt of NE Arctic Russia is widely believed to be the result of the collision of the Arctic AlaskaChukotka microplate with Eurasia, the South Anyui Zone (SAZ) representing the corresponding suture (Fig. 1) (e.g. Parfenov, 1984,1991, 1994; Natal'in et al., 1999; Bogdanov and Til'man, 1992; Seslavinskiy, 1979; Sokolov et al., 2002; Bondarenko et al., 2003). The current most popular plate tectonic model for this collision involves the rotation and southward motion of the Arctic Alaska-Chukotka plate during the opening of the Amerasian Basin of the Arctic Ocean (e.g. Rowley and Lottes, 1988; Grantz et al., 1990; Lawver et al., 2002). The timing of deformation in the Anyui-Chukotka foldbelt and in the SAZ is thus critical to plate models for the Arctic but remained uncertain due to limited geochronologic constraints. In this paper we summarize field observations and structural data that bear on the relative timing of deformational events in the Bilibino region of the Russian Arctic and present new geochronologic and thermochronologic data that help place more reliable age brackets on both deformation and magmatism across this region. An extension-related deformational event that took place during significant input of magma into the crust between $\sim 120$ and $108 \mathrm{Ma}$ is recognized and described for the first time. We suggest that this magmatism could represent a continental manifestation of the rifting process that formed the Amerasian Basin of the

Published by Copernicus Publications on behalf of the European Geosciences Union. 


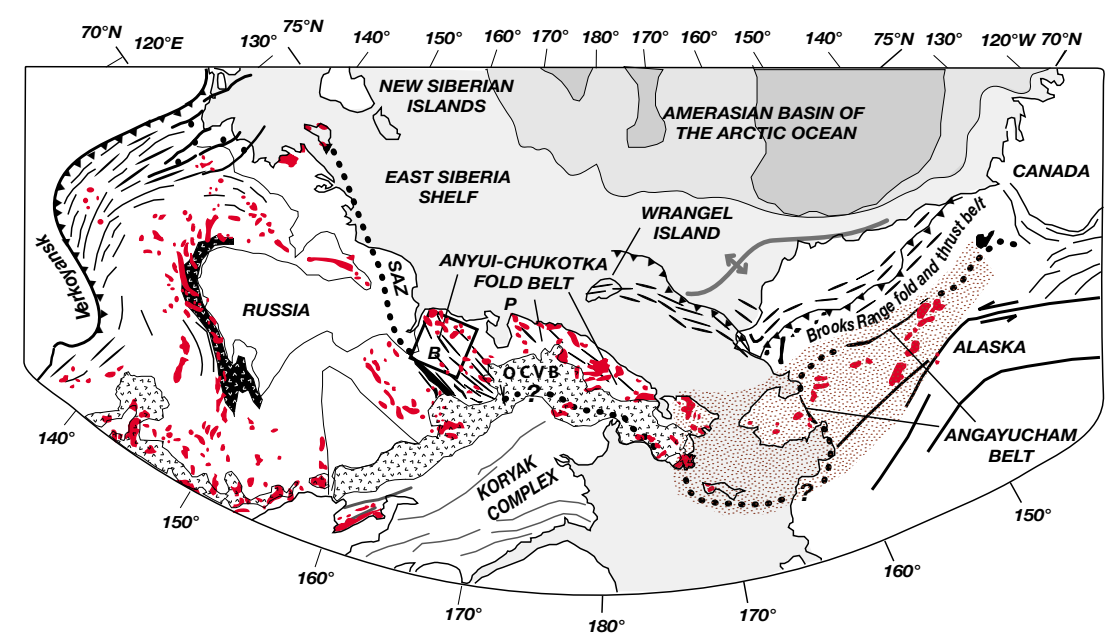

Fig. 1. Index map of the main Mesozoic structural belts of NE Arctic Russia and northern Alaska showing the geographic extent of the Anyui-Chukotka fold belt (also referred to as the Chukotka fold belt) and location of the South Anyui suture zone (SAZ) relative to the Brooks Range fold and thrust belt, Colville foreland basin (north of the Brooks Range) and ophiolitic Angayucham belt in Alaska and the fold belt and ophiolites of the Verkhoyansk belt, Russia. Plutons are shown in red (grey) and Okhotsk-Chukotsk volcanic belt (OCVB) with a "v" pattern. Quaternary deposits cover the SAZ between Chukotka and the New Siberian Islands and the OCVB covers the SAZ between western Chukotka and Alaska; its inferred continuation beneath both regions is shown by a heavy dotted line. Known regions of very high Late Cretaceous extensional strain in the Bering Strait region are shown by a light grey pattern (after Amato and Miller, 2004). Location of Fig. 2 is shown by square outline near the town of Bilibino (B). P is town of Pevek. Modified from Miller et al. (2006).

Arctic Ocean. By inference, collision-related deformation that pre-dates this magmatic event, stratigraphically dated as Late Jurassic to Early Cretaceous, would have occurred prior to the rifting and opening of the Arctic Ocean rather than during rifting.

\section{Geologic setting}

A sedimentary succession that ranges in age from at least the Devonian to the Jurassic, succeeded by Late Jurassic to Early Cretaceous syn-orogenic sandstones are the main stratigraphic units involved in folding and thrusting in the Anyui-Chukotka foldbelt (Fig. 2). Precambrian basement is inferred to underlie Devonian strata, but the closest mapped and dated exposure of crystalline basement rocks is on Wrangel Island where they are known to be $600-700 \mathrm{Ma}$ (Kosk'ko et al., 1993). The Devonian to Mississippian section consists of mixed clastics and carbonates and is succeeded by a Carboniferous to Permian (?) carbonate platform sequence. The lower Triassic is notable in that it contains abundant gabbroic dikes and sills. These are the manifestation a rifting event that led to the foundering of the carbonate platform and the development of deep-water basins that accumulated thick siliciclastic turbidite sequences throughout the Mesozoic (e.g. Gel'man, 1963a; Tuchkova et al., 2006, 2007; Tuchkova et al., this volume).

Stratigraphic constraints on the age of deformation in the Anyui-Chukotka fold belt are provided by Jura-Cretaceous syn-orogenic strata. Uplift of source regions began in the latest Jurassic and syn-orogenic sediments were deposited northwards across the SAZ as a consequence of uplift and deformation (e.g. Bondarenko et al., 2003; Miller and Verzhbitsky, this volume; Miller et al., 2004, 2006, 2009). Syn-orogenic strata are as young as Early Cretaceous (Valanginian) based on scattered buchia fossil localities across Chukotka (Sosunov and Til'man, 1960) and are in turn folded and involved in thrust-faulting (Baranov, 1996; Akimenko, 2000) (Fig. 2). In the SAZ proper, Dovgal (1964) describes strata with fossils as young as Hauterivian-Barremian that are involved in at least the latest stages of deformation (see also summary in Bondarenko et al., 2003). These interpretations of the stratigraphic constraints on the age of deformation differ from earlier views summarized in Nokleberg et al. $(1994,1998)$ who categorized all of what we term "syn-orogenic" deposits as posttectonic, "overlap" sequences.

Cretaceous plutons are widespread across the AnyuiChukotka foldbelt, but existing K-Ar data have large errors and thus the age range of magmatism is only poorly constrained. During 1:200000 scale geologic mapping of the region (e.g. Sadovskiy and Gel'man, 1970; Belik and Sosunov, 1969; Chasovitin and Shpetnyi, 1964; Sosunov and Til'man, 1960), the ages of plutonic rocks were determined by the K-Ar method using whole rock and mineral separates. According to reported data, the age of the oldest, moderately alkalic (syenitic) part of the biggest intrusive complex in the region, the Lupveem batholith, is $123 \mathrm{Ma}$ (Sadovskiy and Gel'man, 1970). The younger granitic parts of the complex have reported K-Ar ages of $100 \mathrm{Ma}$ and pegmatite dikes 


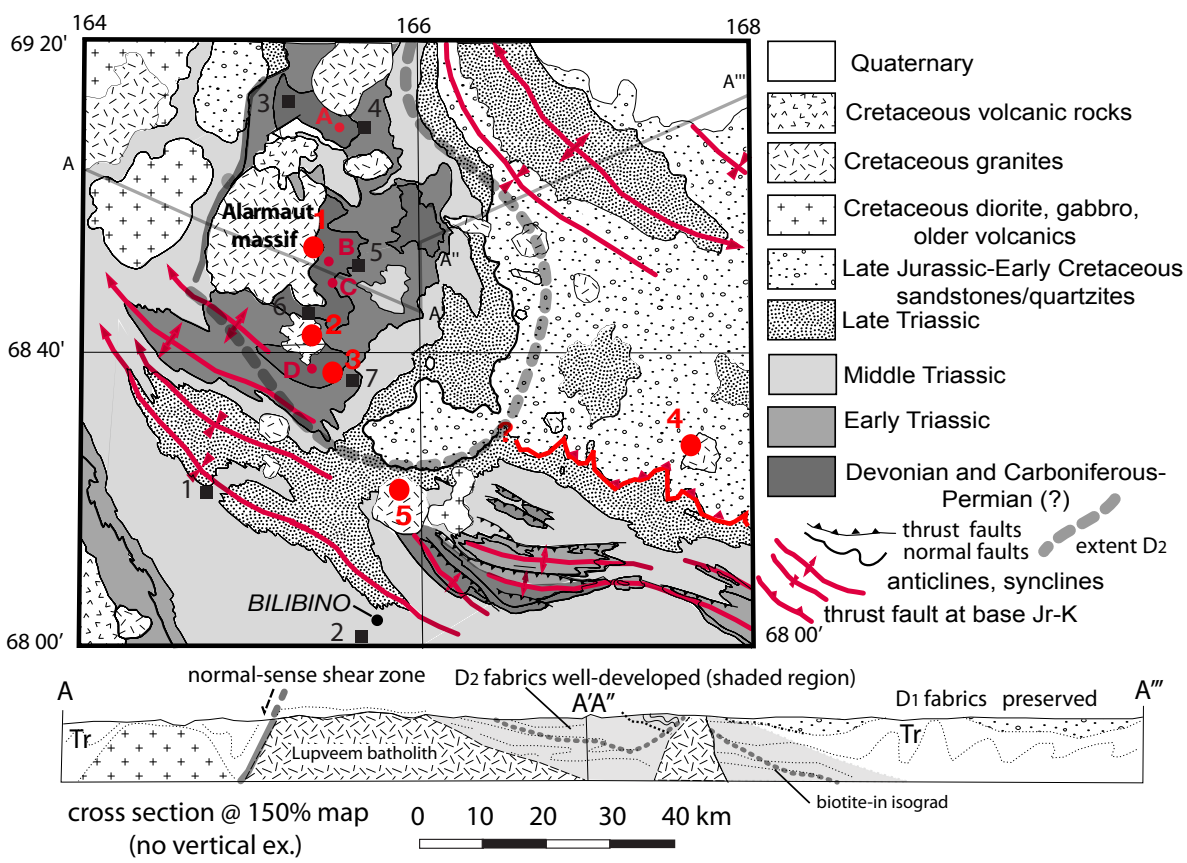

Fig. 2. Simplified geologic map and cross-section of the region north of Bilibino, Chukotka, For location of map see Fig. 1. Based on 1:200 000 scale geologic maps and sections of Gel'man and Sadovskii (1967), Belik and Sosunov (1969), Chasovitin and Shpetnyi (1964) and Sosunov and Tilman (1960). Red dots and numbers correspond to the approximate location of dated samples dated with the U-Pb method (for exact sample locations see Table 1). Locality 1 includes samples ELM03 CH16.1 and CH16.2 of the Lupveem batholith of the Alarmaut Massif. Locality 2 is the Bystrinski pluton and sample JT04-14. Locality 3 is sample ELM03 CH12.1 from a small foliated granitic sill-like body. Locality 4 is the Kelilvun pluton and sample JT04-46. Locality 5 is the Pyrkanai pluton and sample JT04-4. Sample AN100 lies south of the map area within the South Anyui Zone (see Fig. 6). Red dots labeled A-D are ${ }^{40} \mathrm{Ar} /{ }^{39} \mathrm{Ar}$ analyses of metamorphic biotite (Fig. 14 and Table 3). Squares with numbers are localities where outcrop-scale structures were measured and compiled in Fig .3.

have reported K-Ar ages of $90 \mathrm{Ma}$, but none of these analyses have the precision of modern analyses that is needed to establish good age brackets on intrusive histories.

The Okhotsk-Chukotsk Volcanic Belt (OCVB) is often cited as the younger age bracket for deformation because it unconformably overlies the rocks of the SAZ and its regional map continuity is not obviously broken by the SAZ (Fig. 1). However, the oldest volcanic rocks of the OCVB are now known to be mostly younger than $90 \mathrm{Ma}$ based on ${ }^{40} \mathrm{Ar} /{ }^{39} \mathrm{Ar}$ and U-Pb dating (e.g. Lane et al., 1998; Hourigan and Akinin, 2004; Akinin and Khanchuk, 2005; Akinin et al., 2006; Tichomirov et al., 2006), thus the importance of precisely dating the older granitoid plutons is clear: These are the oldest rocks that cut structures related to the AnyuiChukotka foldbelt and the SAZ and therefore have the potential to provide tighter age brackets for D1 deformation (Figs. 1 and 2).

Three field seasons (2002, 2003 and 2004) were spent in the South Anyui Zone and in the Anyui-Chukotka foldbelt in order to investigate the granitoid intrusions in this region and their relationship to country rock deformation. Below we summarize the results of these studies, which provide a geologic and tectonic context for the geochronologic and thermochronologic data presented in this paper.

\section{Structural geology}

Triassic strata underlie most of the region studied (Fig. 2) and are folded into tight W-NW trending map-scale folds with steep limbs. (Figs. 2 and 3a-d). The rocks exhibit a pronounced, but variably developed axial plane cleavage that formed at low greenschist-facies conditions (chlorite and white mica stable). Outcrop-scale folds and beddingcleavage intersection lineations are parallel to mapped regional fold axes (Fig. 3a-d). This deformation $\left(D_{1}\right)$ is the earliest penetrative deformation recorded in the rocks and resulted from shortening in a NE-SW direction. The steep dips of bedding, tight outcrop and map-scale folds, and moderate to steep axial plane cleavage indicate these structures record significant horizontal shortening (Fig. 2).

The region of relatively high mountains underlain by higher grade metamorphic rocks, intruded by the Lupveem batholith, is termed the Alarmaut massif (Fig. 2). With increasing proximity to the Lupveem batholith, earlier deformational fabrics become strongly overprinted by a second deformation $\left(D_{2}\right)$ characterized by a shallowly dipping foliation $\left(S_{2}\right)$ (Figs. 3e and $4 \mathrm{a}-\mathrm{e}$ ). This event recumbently folds the earlier steeply-dipping axial plane cleavage and original bedding (Fig. $4 \mathrm{c}$ and e). At the outcrop and thinsection scale, unit contacts are transposed to sub-horizontal 


\section{First deformational event D1}
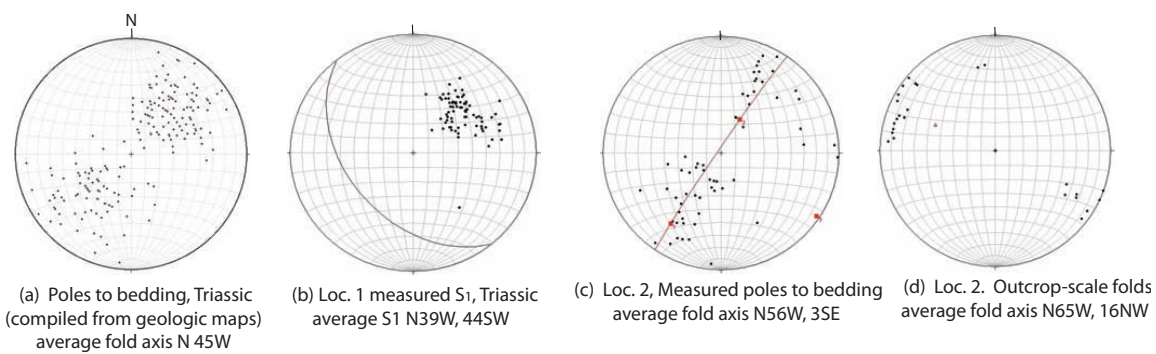

Second deformational event D2
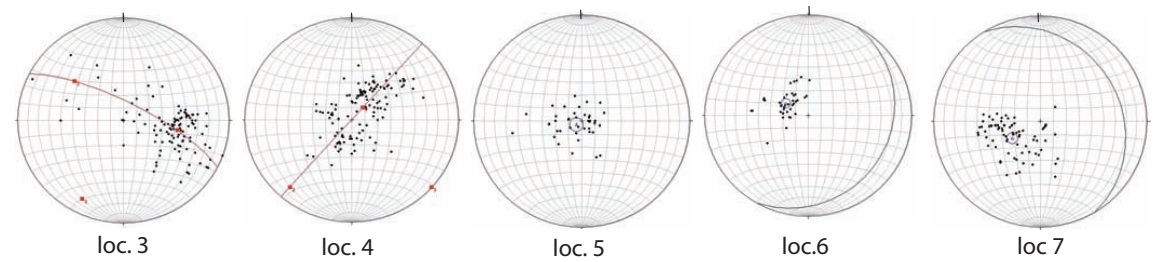

(e) Poles to gently dipping S2 foliation at various localities
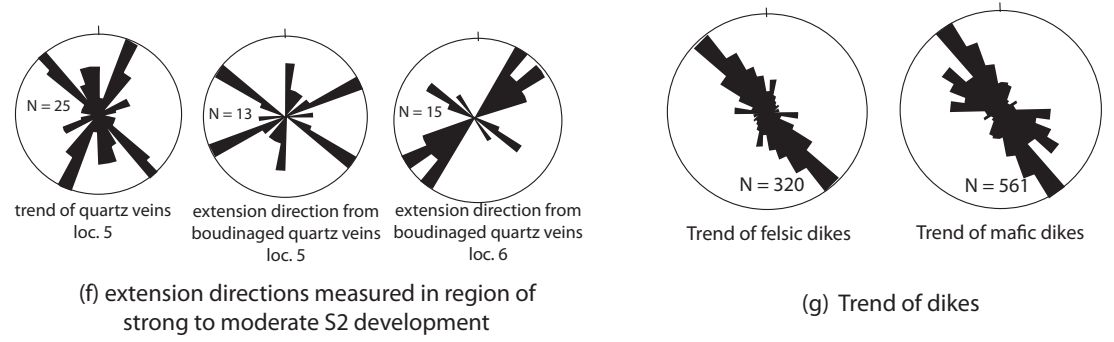

(g) Trend of dikes

Fig. 3. Selected structural data for the Bilibino region. Stereonet plots are equal angle lower hemisphere projections. (a) Poles to measured bedding in and around Bilibino and north to the Alarmaut massif compiled from strikes and dips on 1:200000 scale geologic maps by Gel'man and Sadovskii (1967), Belik and Sosunov (1969), Chasovitin and Shpetnyi (1964) and Sosunov and Tilman (1960). (b) Poles to measured $S_{1}$ foliation at structure locality 1 (Fig. 2). (c) Poles to measured bedding at structure locality 2 (Fig. 2). (e) Measured outcrop-scale fold axes at structure locality 2 (Fig. 2). (e) Poles to measured $S_{2}$ foliation east and north of Alarmaut massif, keyed to structure localities shown on Fig. 2. Red great circles show warping/open folding of $S_{2}$. Black great circles are average orientation of $S_{2}$. (f) Measured quartz veins at structure locality 5 (Fig. 2) and extension directions measured orthogonal to boudin axes of stretched quartz veins at structure localities 5 and 6. (g) Trend of mafic and felsic dikes compiled from mapped dikes shown on 1:200,000 scale geologic maps by Gel'man and Sadovskii (1967), Belik and Sosunov (1969), Chasovitin and Shpetnyi (1964) and Sosunov and Tilman (1960). Inferred extension direction is orthogonal to these, or NE-SW.

by tight to isoclinal folds (Fig. 4a). $S_{2}$ is pervasive for a broad distance to the east of the Alarmaut Massif (Fig. 2). The highest strains associated with $S_{2}$ occur along the eastern contact of the Lupveem batholith. Here, all rock units and their contacts are subhorizontal, transposed into parallelism with the metamorphic foliation (Fig. 4d). Where contrasts in rock rheology exist, as between mafic diabase dikes and sills of Permo-Triassic age and Carboniferous-Permian limestones (marbles), subhorizontal detachment surfaces are present which omit section (Fig. 4a). The subhorizontal attitude of foliation and the drastic thinning of units lead us to the interpretation that these fabrics are related to horizontal extension (and vertical thinning). However, stretching directions defined by mineral elongation lineations are only locally developed. We measured boudinaged quartz veins and cross-cutting quartz veins that yielded variable extension di- rections ranging from NW-SE to to NE-SW (Fig. 3f). The trend of mafic and felsic dikes associated with granitoid intrusions were measured from 1:200000 scale geologic maps of the region and are fairly consistently oriented NW-SE, indicating a NE-SW extension direction during magmatism (Fig. 3g).

Near the Lupveem batholith, $S_{2}$ is a pervasive metamorphic foliation defined by the new growth and alignment of metamorphic minerals such as chlorite, muscovite, biotite and actinolite. (Fig. 4d). Away from the batholith, $S_{2}$ becomes a spaced crenulation cleavage (Fig. 4e). The metamorphic grade of the country rocks is highest in the southeastern part of the Alarmaut massif where we observed sillimanite together with andalusite in amphibolite-facies country rocks of the Bystrinki pluton (Locality 2, Fig. 2). Based on the close spatial relationship between the margins of the 


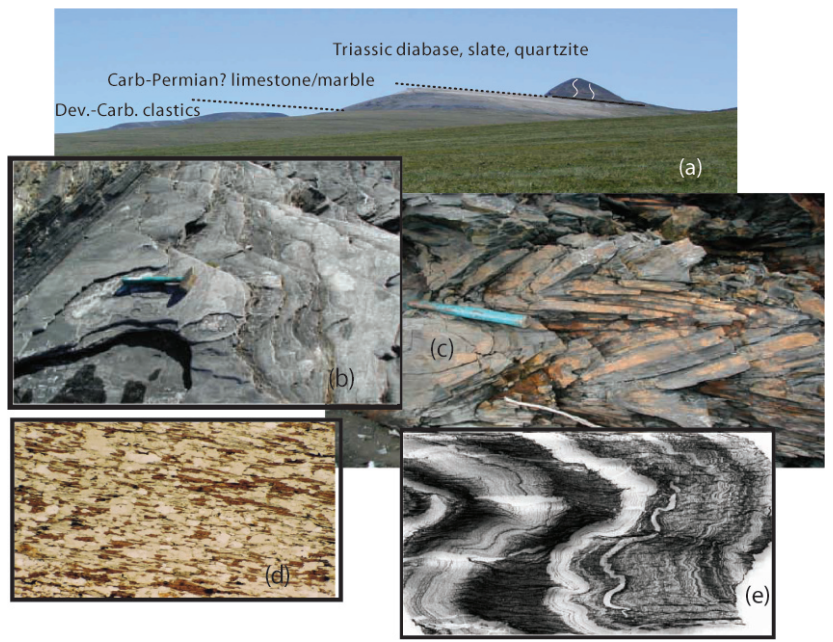

Fig. 4. $D_{2}$ deformational event: (a) View looking to the $\mathrm{N}$ of exposures on the east side of the Alarmaut massif showing pronounced gently east dipping foliations and thinned stratigraphic units, including the omission-fault-bound base of the Triassic. Note that Triassic units above this contact are steep and truncated at the contact. (b) Carboniferous-Permian marbles, strongly foliated and lineated. Hammer and lineations are oriented NW-SE, view is looking NE. Looking NE. (c) Outcrop-scale recumbent folds of bedding and $S_{1}$ (which is weak and at a low angle to bedding in this location) in Triassic strata. Axial planes of folds are $S_{2}$. (d) Thin section of sample ELM03 CH27. 2, Devonian-Carboniferous clastic rocks near Alarmaut massif showing strong foliation $\left(S_{2}\right)$ defined by biotite. Field of view about $3 \mathrm{~mm}$ across, plane light. (e) Thin-section of $\mathrm{S}_{2}$ crenulating and folding earlier $S_{1}$ and bedding in Triassic slates. Scanned thin-section is about $3 \mathrm{~cm}$ in length and is cut perpendicular to $S_{2}$ and intersection of $S_{1}$ and $S_{2}$.

Lupveem batholith and the metamorphic grade and minerals that define $S_{2}$ (Fig. 2, cross-section), it is reasonable to infer that the Lupveem batholith was at least partially emplaced during $D_{2}$. However, most of the contacts we observed between country rocks and plutons and their associated dikes are sharply cross-cutting. Exceptions include small sill-like bodies of granitic to aplitic composition near the southern edge of the Alarmaut massif. Some of these bodies are variably foliated with fabrics that are subparallel to $D_{2}$ fabrics $\left(S_{2}\right)$ of the metasedimentary country rocks (e.g. sample ELM03-CH12.1, Fig. 2, Locality 3).

The Lupveem batholith consists mostly of granite and granodiorite, but minor syenite and gabbro have been mapped within the massif (Bondarenko and Luchitskaya, 2003; Sadovskiy and Gel'man, 1970). We investigated the mapped contact between the granite and granodiorite phases of the batholith on the eastern side of the massif, and determined that the granitic phase is younger as it includes xenoliths of the granodiorite along the contact between the two phases (Fig. 5a). Porphyritic granite dikes, with tabular K-feldspars phenocrysts (Fig. $5 b$ ) cut both the granite and the granodiorite of the Lupveem batholith and are the youngest intrusive phases recognized in the batholithic complex.
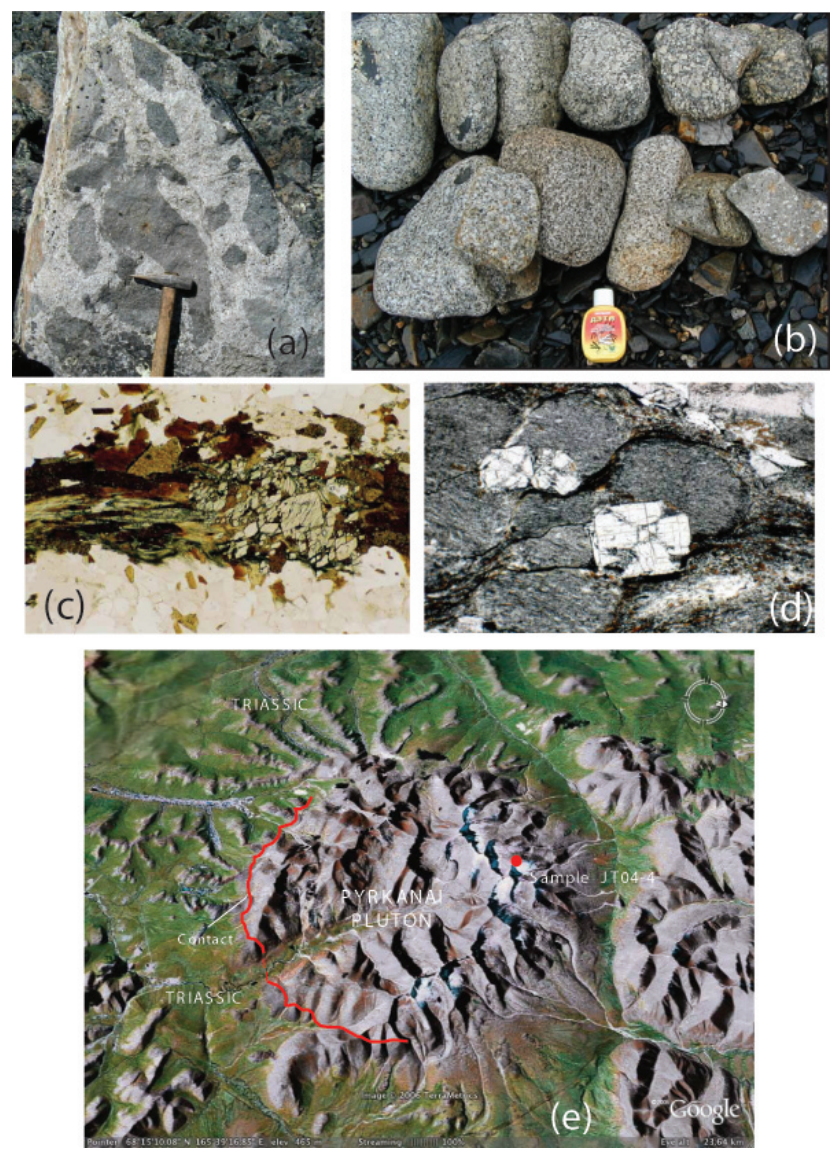

Fig. 5. (a) Fragments of granodiorite in the granitic phase of the Lupveem batholith (Fig. 2, loc. 1). (b) Close up of plutonic and dike rock types that range from equigranular to porphyritic. (c) Thin section photomicrograph of sillimanite-biotite bearing schist, country rocks to Bystrinski pluton adjacent to loc. CH04 JT-14 (Fig. 2). Field of view about $3 \mathrm{~mm}$. (d) Thin section photomicrograph of andalusite and inclusion-ridden chloritoid, field of view is about $3 \mathrm{~mm}$. (e) Google Earth view of Pyrkanai pluton and sample locality CH04-JT4 (Fig. 2). Contact with Triassic country rocks highlighted by red line. Note that north is to the right.

The Bystrinski pluton along the southeastern flank of the Alarmaut massif (Fig. 2, Locality 2) intrudes Triassic slates and quartzites and Permo-Carboniferous marbles, all metamorphosed to amphibolite facies with sillimanite and andalusite present in pelitic protoliths (Fig. 5c) (Gel'man, 1963b). Small bodies of variably foliated granite intrude country rocks on the southern side of the Bystrinski pluton and were sampled for geochronology because their intrusion appears to be syntectonic with the development of $D_{2}$ fabrics.

The isolated Kelilvun and Pyrkanai plutons (Localities 4 and 5, Fig. 2) to the east and south of the Lupveem batholith are approximately circular in map view as are many of the other plutons that intrude the Anyui-Chukotka fold belt (Fig. 2). They range in composition and texture from 
medium-grained, biotite-bearing equigranular granites and granodiorites to porphyritic granites with large tabular Kfeldspar phenocrysts (Fig. 5b). The Pyrkanai pluton intrudes Upper Triassic deposits, while the Kelilvun granite intrudes Lower Cretaceous syn-orogenic siliciclastic rocks. The mapped contacts of the plutons are generally moderately to steeply dipping. Country rocks are domed around the intrusive contacts of circular plutons and often have a strong (and steep) foliation developed immediately adjacent to the contact (Fig. 5f). Chloritoid \pm andalusite are syn- to posttectonic (Fig. 5d). Unlike the Lupveem batholith, the isolated Pyrkanai and Kelilvun plutons are not associated with the shallowly dipping S2 foliation or with regional metamorphism but may also represent shallower exposures (Mesozoic country rocks as opposed to Paleozoic). Their contact aureoles and their shapes in map pattern, however, clearly show that they truncate the widely developed steep S1 foliation and associated folds (Fig. 2).

The southernmost dated granite (Sample AN100) lies within the SAZ (Fig. 6). The dominant map-scale structures developed in the SAZ are parallel to those of the AnyuiChukotka fold belt (e.g. Figs. 1, 2 and 6). Despite the interest and plate tectonic significance of this suture zone, it is still only very poorly understood (e.g. Sokolov et al., 2002; this volume). The region of the SAZ is topographically low-lying and outcrop-scale structures are difficult to measure because of extensive frost-heaving. Based on geologic mapping by Dovgal (1964) (Fig. 6), map-scale structures involving Triassic, Jurassic and Cretaceous strata have regional orientations parallel to those of the Anyui-Chukotka foldbelt and bedding mostly dips steeply (Fig. 6). In addition, zones of shearing or high strain deformation have been mapped parallel to these regional trends (Fig. 6). The contact relations of the pluton are not exposed in outcrop, but the map-scale structures and zones of high deformation characterizing the SAZ are truncated by the circular outline of the pluton and its contact metamorphic aureole (Fig. 6).

In summary, based on existing geologic mapping of the region, our field observations and measurement of outcropscale structures, the granitoid plutons in this part of the Anyui-Chukotka fold belt clearly post-date $D_{1}$ and those of the Lupveem batholith intruded and provided the heat source for metamorphism during $D_{2}$. This younger deformation tightly to isoclinally recumbently refolds the earlier $D_{1}$ fabrics and is associated with metamorphism ranging from amphibolite (locally) to greenschist facies across a broad region around and to the east of the batholith (Fig. 2).

Across the area studied (Fig. 2), a significant regional unconformity was developed prior to the deposition of volcanic rocks of the OCVB. This unconformity bevels $D_{1}$ and $D_{2}$ structures and has eroded the cover rocks above the granitoid plutons. Based on the metamorphic assemblages developed in the contacts of the plutons (andalusite stable), there was likely no more than $\sim 12 \mathrm{~km}$ but likely at least several kilometers of structural section removed by erosion. This unconfor- mity was developed sometime between $108.5 \pm 2.7 \mathrm{Ma}$, the youngest pluton that we have dated (Albian), and $87 \mathrm{Ma}$, the oldest nearly flat-lying volcanic rocks of the OCVB (Coniacian) (Hourigan and Akinin, 2004; Akinin and Khanchuk, 2005; Akinin et al., 2006; Tichomirov et al., 2006).

\section{Geochronology}

\subsection{U-Pb analyses}

$\mathrm{U}-\mathrm{Pb}$ analysis has become one of the most reliable and widely used forms of geochronology to determine crystallization ages for igneous rocks. Zircon is most often found as an accessory mineral in felsic to intermediate composition igneous rocks, and has a closure temperature of greater than $900^{\circ} \mathrm{C}$ (Mezger and Krogstad, 1997; Dahl, 1997), such that granulite-facies conditions are usually required to reset its $\mathrm{U}$ $\mathrm{Pb}$ systematics. Igneous rocks may incorporate preexisting zircons by melting a source material that contains zircons, or by assimilation of wall rocks, and these inherited grains can survive melting in the source region. Zircons can develop age domains and commonly rims grow on pre-existing cores (Mezger and Krogstad, 1997). Cathodoluminescence (CL) of zircon grains is carried out before age analysis in order to reveal the internal structure of the grains, including cores and rims. Sphene $\left(\mathrm{CaTiOSiO}_{4}\right)$ is also a common accessory mineral in granitoids and mafic rocks with high $\mathrm{Ca} / \mathrm{Al}$ ratios (Frost et al., 2000). It can incorporate both $\mathrm{U}$ and $\mathrm{Pb}$ into its crystalline structure (Frost et al., 2000), and U-Pb analyses commonly show a mixture of radiogenic and common $\mathrm{Pb}$ that fall along a discordia line when plotted on a concordia diagram. Sphene has a closure temperature of $>700^{\circ} \mathrm{C}$ (Cherniak, 1993; Verts et al., 1996; Frost et al., 2000) which for granitic rocks may be close to the temperature of crystallization. If a sphene-bearing pluton cools promptly after intrusion, then the U-Pb ages of the sphene will reflect a crystallization age. However, if the pluton cools slowly, or remains at depth after intrusion, then the sphene will reflect a cooling age through the $\sim 700^{\circ} \mathrm{C}$ isotherm.

All zircons and sphenes from this study were analyzed with the U-Th- $\mathrm{Pb}$ method utilizing the Sensitive High Resolution Ion Microprobe - Reverse Geometry (SHRIMP-RG) at the USGS-Stanford Microanalytical Center at Stanford University. All of the final ages reported here were assigned using the weighted mean averages of ${ }^{207} \mathrm{~Pb}$-corrected ${ }^{206} \mathrm{~Pb} /{ }^{238} \mathrm{U}$ ages, rather than concordia intercept ages, because most of the analyses do not show a linear trend when plotted on concordia diagrams. However, Figures 612 are concordia plots of the uncorrected data after Tera and Wasserburg (1974). The main advantages of using $\mathrm{U}-\mathrm{Pb}$ concordia diagrams to interpret geochronologic data are: 1) the ability to detect disturbances to the isotopic systematics of a mineral (i.e., discordance), 2) the ability to detect $\mathrm{Pb}$ loss, and 3) the assessment of any common 


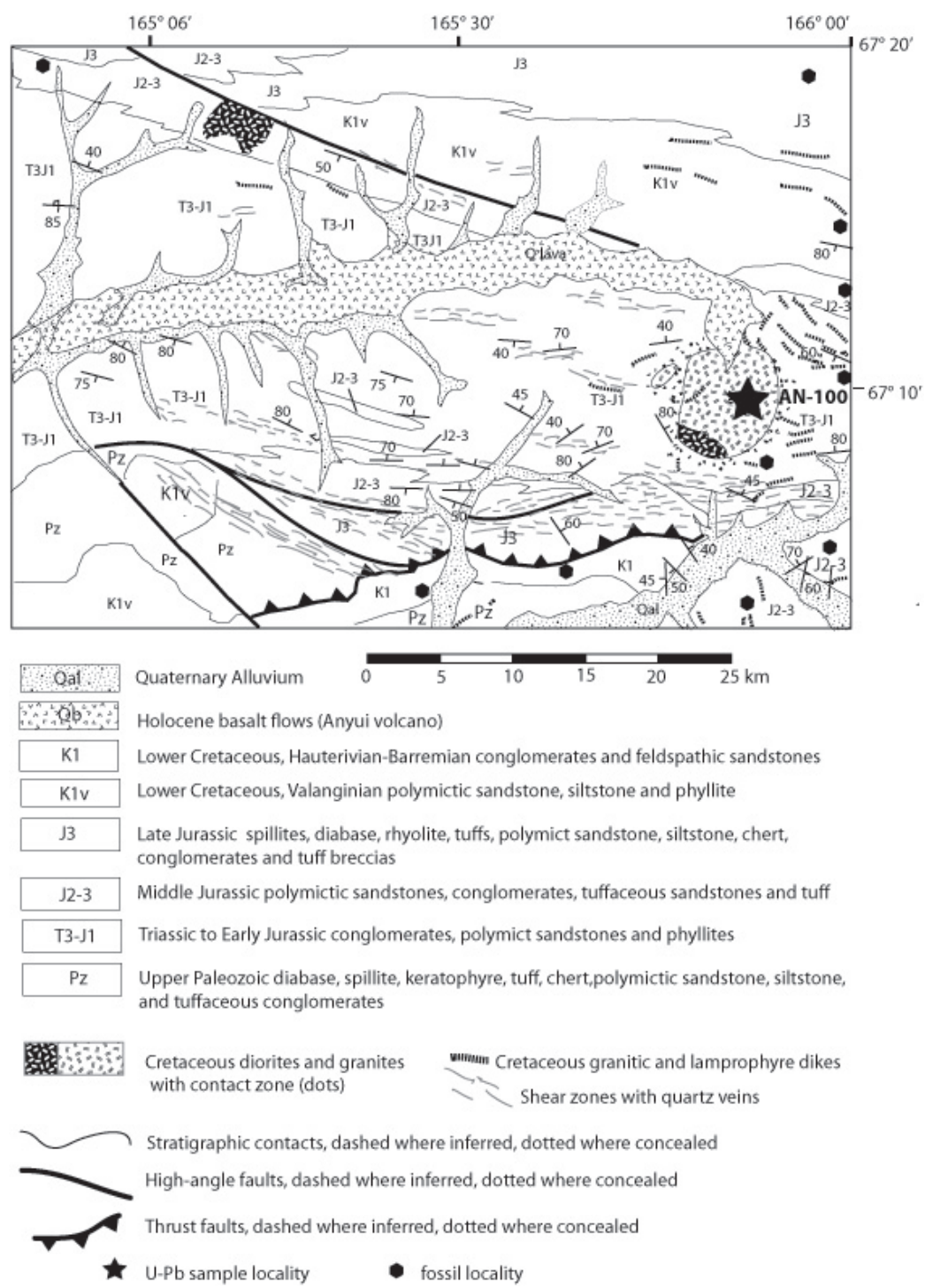

Fig. 6. Geologic map of a portion of the SAZ showing the location and tectonic setting of sample AN-100. After Dovgal (1964).

$\mathrm{Pb}$ component. Concordance is interpreted to indicate a closed isotopic system with a single crystallization age. Table 1 lists the samples analyzed and their locations. Analytical techniques are described at the end of Table 2.

\subsubsection{Lupveem batholith}

Sample ELM03-CH16.2 is from a medium-grained, equigranular, hornblende/biotite-bearing granodioritic phase of the Lupveem batholith of the Alarmaut massif (Locality 1, Fig. 2, Table 1). Based on field relationships (Fig. 5), we interpret this unit to be older than the main granitic phase of the batholith. K-Ar ages reported from this batholith are $103 \pm 2.5$ and $114 \pm 6 \mathrm{Ma}$ (Gel'man, 1963b, in Akinin and Kotlyar, 1997), which lie within error of biotite ages we report for the cooling of metamorphic country rocks (below). Zircons from this sample are euhedral, equant to bladed, and show regular oscillatory zoning in CL imaging (Fig. 8). Cores were visible in some grains, but were subtle and were not analyzed. Ten analyses from this sample are shown in Fig. 8 on a Tera-Wasserburg concordia diagram. Nine of the analyses are concordant within analytical error, with only one analysis plotted slightly above concordia. All ten analyses define a weighted mean age of 115.7 $\pm 1.7 \mathrm{Ma}(\mathrm{MSWD}=0.48$; Table 2), which is interpreted as a crystallization age for this phase of the Lupveem batholith.

Sample ELM03-CH16.1 is from the most voluminous phase of the Lupveem batholith; an equigranular to porphyritic (K-feldspar) biotite bearing granite (Fig. 2, Table 1). Field relations indicate that this phase of the batholith intrudes the granodioritic phase, because the latter is present as 
Table 1. Sample localities and summary of geochronologic data.

\begin{tabular}{|c|c|c|c|c|c|c|}
\hline $\begin{array}{l}\text { Map } \\
\#\end{array}$ & $\begin{array}{l}\text { Sample } \\
\#\end{array}$ & $\begin{array}{l}\text { Latitude } \\
\text { longitude }\end{array}$ & Rock type & Location & $\begin{array}{l}\text { Zircon: weighted mean } \\
\text { average of }{ }^{207} \mathrm{~Pb} \text {-corrected } \\
{ }^{206} \mathrm{~Pb} /{ }^{238} \mathrm{U} \text { age }\end{array}$ & $\begin{array}{l}\text { Sphene: weighted mean } \\
\text { average of }{ }^{207} \mathrm{~Pb} \text {-corrected } \\
{ }^{206} \mathrm{~Pb} /{ }^{238} \mathrm{U} \text { age }\end{array}$ \\
\hline 1 & ELM03 CH16.1 & $68.982694,165.396778$ & granite, main phase & Lupveem batholith & $115.7 \pm 1.7 \mathrm{Ma}(0.48)^{*}$ & *MSWD \\
\hline 1 & ELM03 CH16.2 & $68.890722,165.399583$ & granodiorite & Lupveem batholith & $112.0 \pm 1.7 \mathrm{Ma}(2.13)$ & \\
\hline 2 & JT04-14 & $68.74075,165.333889$ & granite/granodiorite & Bystrinski pluton & $116.9 \pm 2.5 \mathrm{Ma}(1.8)$ & $106.4 \pm 2.5(0.82)^{*}$ \\
\hline 3 & ELM03 CH12.1 & $68.623944,165.5035$ & granite/aplite dike & $\mathrm{S}$ side Bystrinski pluton & $112.8 \pm 1.7 \mathrm{Ma}(1.17)$ & \\
\hline 4 & JT04-46 & $68.762778,166.969444$ & granite/granodiorite & Kelilvum pluton & $114.7 \pm 1.8 \mathrm{Ma}(0.75)$ & \\
\hline 5 & JT04-4 & $68.367778,165.838333$ & K-spar porphyry granite & Pyrkanai pluton & $112.4 \pm 1.6 \mathrm{Ma}(0.66)$ & $110.6 \pm 1.7(1.04)$ \\
\hline \multirow[t]{2}{*}{ Fig. 6} & AN 100 & $67.157778,165.781944$ & granite/granodiorite & South Anyui Zone & $108.5 \pm 2.7 \mathrm{Ma}(0.82)$ & \\
\hline & & & & & Best $40 \mathrm{Ar} / 39 \mathrm{Ar}$ plateau age & \\
\hline A & ELM 03 CH27.2 & $69.15794,165.18781$ & Dev.-Carb. mu-bi schist & E side Alarmaut Massif & $107.7 \pm 1.2$ & \\
\hline B & ELM 03 CH14.4 & $68.87578,165.6389$ & Dev.-Carb. mu-bi schist & Alarmaut Massif & $104.2 \pm 1.3^{* *}$ & ** age of high $\mathrm{T}$ step \\
\hline $\mathrm{C}$ & ELM 03 CH14.1 & $68.85447,165.63889$ & Dev.-Carb. mu-bi schist & Alarmaut Massif & $109.3 \pm 1.2$ & \\
\hline $\mathrm{D}$ & ELM 03 CH12.6 & $68.63153,165.48492$ & Dev.-Carb. mu-bi schist & Alarmaut Massif & $103.0 \pm 1.8 \mathrm{Ma}$ & \\
\hline
\end{tabular}
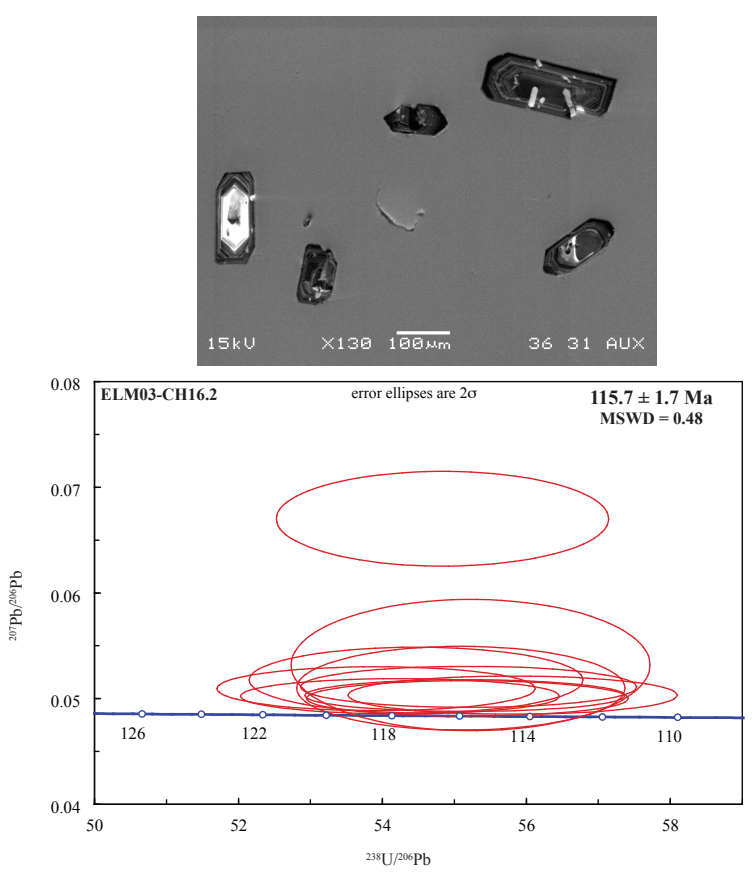

Fig. 7. Sample ELM03-CH16.2: granodioritic phase of the Lupveem Batholith. Top: Cathodoluminescence image of representative zircons from this sample. Bottom: Tera-Wasserburg concordia diagram showing uncorrected U-Pb SHRIMP ages. Error ellipses are shown at two sigma uncertainty. Final age assigned using the weighted mean ${ }^{207} \mathrm{~Pb}$-corrected ${ }^{206} \mathrm{~Pb} /{ }^{238} \mathrm{U}$ age of all ten analyses.

xenoliths in the younger granite along their contact (Fig. 5a). Zircons from sample ELM03-CH16.1 are slender and euhedral and are either homogeneous in CL or show a crude oscillatory zoning pattern. Sixteen grains were analyzed (Table 2 and Fig. 9). All but one of the analyses were concordant within analytical error. The analyses show some scatter on the Tera-Wasserburg concordia diagram $\left({ }^{207} \mathrm{~Pb}\right.$ corrected ${ }^{206} \mathrm{~Pb} /{ }^{238} \mathrm{U}$ ages range from $106.2-119.7 \mathrm{Ma}$ ). Thir-

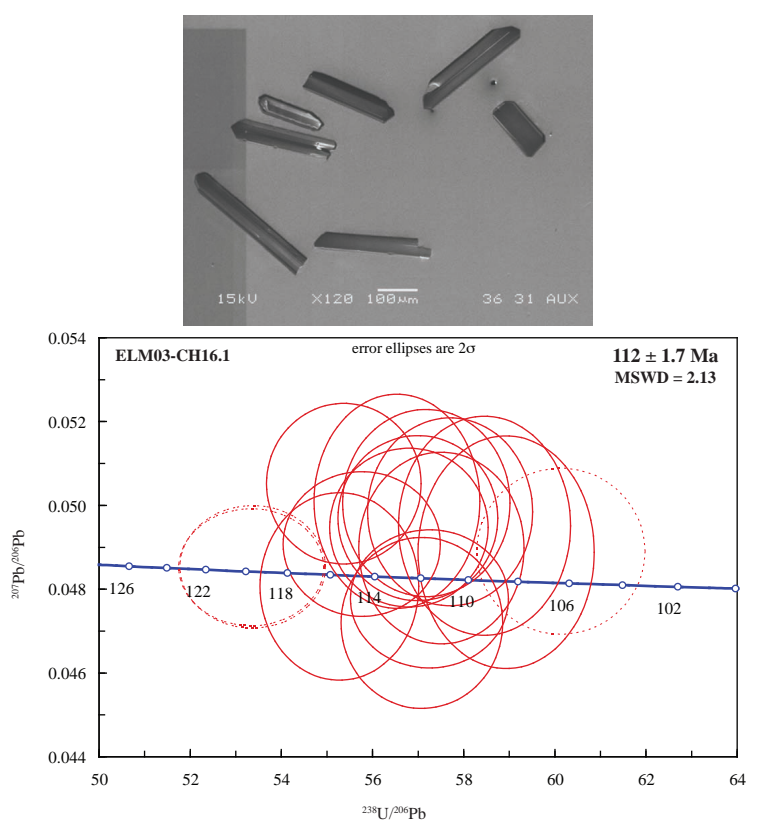

Fig. 8. Sample ELM03-CH16.1: granitic phase of the Lupveem Batholith. Top: Cathodoluminescence image of representative zircons from this sample. Bottom: Tera-Wasserburg concordia diagram showing uncorrected U-Pb SHRIMP ages. Error ellipses are shown at two sigma uncertainty. Final age assigned using the weighted mean ${ }^{207} \mathrm{~Pb}$-corrected ${ }^{206} \mathrm{~Pb} /{ }^{238} \mathrm{U}$ age of thirteen analyses. Three additional analyses are shown in dashed ellipses.

teen of the sixteen analyses were used to produce a weighted mean age of $112 \pm 1.7 \mathrm{Ma}$ (MSWD=2.13), which we interpret as the crystallization age for the granitic phase of the Lupveem batholith. The three analyses not used to calculate the weighted mean age were rejected due to high U-content. Including them in the weighted mean age calculation provides an age of $112.5 \pm 1.9$ (MSWD=6.6). 
Table 2. Isotopic data used to construct U-Pb concordia diagrams. Values shown in italics not used to calculate ages. In the "Analysis" column: final $\mathrm{R}=$ rim, $\mathrm{C}=$ core.

\begin{tabular}{|c|c|c|c|c|c|c|c|c|c|c|c|c|c|c|}
\hline Analysis & $\begin{array}{l}\% \text { initial } \\
{ }^{206} \mathrm{~Pb}\end{array}$ & $\begin{array}{l}\mathrm{U} \\
(\mathrm{ppm})\end{array}$ & $\begin{array}{l}\text { Th } \\
\text { (ppm) }\end{array}$ & $\begin{array}{l}{ }^{232} \mathrm{Th} / \\
{ }^{238} \mathrm{U}\end{array}$ & $\begin{array}{l}{ }^{207} \mathrm{~Pb} \\
\text { corrected } \\
{ }^{206} \mathrm{~Pb} / \\
{ }^{238} \mathrm{U} \text { Age }\end{array}$ & $\begin{array}{l}1 \text { sigma } \\
\text { error }\end{array}$ & $\begin{array}{l}{ }^{207} \mathrm{~Pb} \\
\text { corrected } \\
{ }^{206} \mathrm{~Pb} / \\
{ }^{238} \mathrm{U}\end{array}$ & $\begin{array}{l}1 \text { sigma } \\
\text { error }\end{array}$ & $\begin{array}{l}\text { Total } \\
238 \mathrm{U} / \\
{ }^{206} \mathrm{~Pb}\end{array}$ & $\begin{array}{l}\% \\
\text { error }\end{array}$ & $\begin{array}{l}\text { Total } \\
{ }^{207} \mathrm{~Pb} / \\
{ }^{206} \mathrm{~Pb}\end{array}$ & $\begin{array}{l}\% \\
\text { error }\end{array}$ & $\begin{array}{l}{ }^{204} \mathrm{~Pb} \\
\text { corrected } \\
{ }^{207} \mathrm{~Pb} / \\
{ }^{206} \mathrm{~Pb}\end{array}$ & $\begin{array}{l}\% \\
\text { error }\end{array}$ \\
\hline \multicolumn{15}{|c|}{ ELM03-CH16.2: Granodioritic phase of the Lupveem Batholith. Weighted mean average of ${ }^{207} \mathrm{~Pb}$-corrected ${ }^{206} \mathrm{~Pb} / 238 \mathrm{U}$ age $=115.7 \pm 1.7 \mathrm{Ma}, \mathrm{MSWD}=0.48$. } \\
\hline ELM03-CH16.2-1 & 0.21 & 2363 & 835 & 0.37 & 115.5 & 1.9 & 0.0181 & 0.0003 & 55.18 & 1.7 & 0.0500 & 1.4 & 0.0488 & 1.6 \\
\hline ELM03-CH16.2-2 & 2.36 & 863 & 126 & 0.15 & 113.8 & 2.0 & 0.0178 & 0.0003 & 54.84 & 1.7 & 0.0670 & 2.7 & 0.0486 & 10.6 \\
\hline ELM03-CH16.2-3 & 0.33 & 1258 & 214 & 0.18 & 115.6 & 2.0 & 0.0181 & 0.0003 & 55.09 & 1.7 & 0.0510 & 3.2 & 0.0483 & 4.2 \\
\hline ELM03-CH16.2-4 & 0.61 & 398 & 116 & 0.30 & 115.0 & 2.1 & 0.0180 & 0.0003 & 55.23 & 1.8 & 0.0532 & 4.8 & 0.0491 & 6.6 \\
\hline ELM03-CH16.2-5 & 0.43 & 770 & 114 & 0.15 & 116.8 & 2.0 & 0.0183 & 0.0003 & 54.47 & 1.7 & 0.0518 & 2.4 & 0.0488 & 4.0 \\
\hline ELM03-CH16.2-6 & 0.35 & 1931 & 577 & 0.31 & 115.3 & 2.0 & 0.0181 & 0.0003 & 55.20 & 1.7 & 0.0511 & 1.5 & 0.0511 & 1.5 \\
\hline ELM03-CH16.2-7 & 0.32 & 1710 & 554 & 0.33 & 118.1 & 2.0 & 0.0185 & 0.0003 & 53.91 & 1.7 & 0.0509 & 1.7 & 0.0475 & 3.2 \\
\hline ELM03-CH16.2-8 & 0.23 & 2424 & 592 & 0.25 & 117.5 & 1.9 & 0.0184 & 0.0003 & 54.24 & 1.7 & 0.0502 & 1.4 & 0.0474 & 2.5 \\
\hline ELM03-CH16.2-9 & 0.25 & 3202 & 1008 & 0.33 & 115.6 & 1.9 & 0.0181 & 0.0003 & 55.15 & 1.6 & 0.0503 & 1.2 & 0.0492 & 1.5 \\
\hline ELM03-CH16.2-10 & 0.25 & 2144 & 719 & 0.35 & 114.2 & 1.9 & 0.0179 & 0.0003 & 55.81 & 1.7 & 0.0503 & 1.5 & 0.0490 & 2.0 \\
\hline \multicolumn{15}{|c|}{ ELM03-CH16.1: Granitic phase of the Lupveem Batholith. Weighted mean average of ${ }^{207} \mathrm{~Pb}$-corrected ${ }^{206} \mathrm{~Pb} /{ }^{238} \mathrm{U}$ age $=112 \pm 1.7 \mathrm{Ma}, \mathrm{MSWD}=2.13$. } \\
\hline ELM03-CH16.1-1 & 0.10 & 2764 & 1111 & 0.42 & 106.2 & 1.3 & 0.0166 & 0.0002 & 60.13 & 1.3 & 0.0489 & 1.7 & 0.0480 & 2.0 \\
\hline ELM03-CH16.1-2.1 & 0.17 & 1500 & 435 & 0.30 & 109.2 & 1.4 & 0.0171 & 0.0002 & 58.46 & 1.3 & 0.0495 & 2.2 & 0.0488 & 2.4 \\
\hline ELM03-CH16.1-3.1 & 0.10 & 3269 & 1507 & 0.48 & 114.5 & 1.4 & 0.0179 & 0.0002 & 55.76 & 1.3 & 0.0491 & 1.4 & 0.0454 & 3.4 \\
\hline ELM03-CH16.1-4.1 & 0.01 & 4278 & 2435 & 0.59 & 119.7 & 1.5 & 0.0187 & 0.0002 & 53.37 & 1.2 & 0.0485 & 1.2 & 0.0485 & 1.2 \\
\hline ELM03-CH16.1-5.1 & 0.01 & 5068 & 3187 & 0.65 & 119.7 & 1.4 & 0.0187 & 0.0002 & 53.34 & 1.2 & 0.0485 & 1.2 & 0.0483 & 1.2 \\
\hline ELM03-CH16.1-6.1 & 0.23 & 2136 & 757 & 0.37 & 111.5 & 1.5 & 0.0174 & 0.0002 & 57.18 & 1.3 & 0.0501 & 1.8 & 0.0501 & 1.8 \\
\hline ELM03-CH16.1-7.1 & -0.06 & 3531 & 1550 & 0.45 & 111.7 & 1.4 & 0.0175 & 0.0002 & 57.25 & 1.2 & 0.0478 & 1.4 & 0.0475 & 1.5 \\
\hline ELM03-CH16.1-8.1 & -0.13 & 2383 & 961 & 0.42 & 112.1 & 1.4 & 0.0175 & 0.0002 & 57.08 & 1.3 & 0.0472 & 1.8 & 0.0459 & 2.2 \\
\hline ELM03-CH16.1-9.1 & 0.15 & 2784 & 1081 & 0.40 & 112.3 & 1.4 & 0.0176 & 0.0002 & 56.80 & 1.3 & 0.0495 & 1.6 & 0.0479 & 2.2 \\
\hline ELM03-CH16.1-10.1 & 0.18 & 2650 & 871 & 0.34 & 111.9 & 1.4 & 0.0175 & 0.0002 & 56.99 & 1.3 & 0.0497 & 1.6 & 0.0493 & 1.7 \\
\hline ELM03-CH16.1-11.1 & 0.09 & 1920 & 698 & 0.38 & 111.0 & 1.4 & 0.0174 & 0.0002 & 57.51 & 1.3 & 0.0490 & 1.9 & 0.0490 & 1.9 \\
\hline ELM03-CH16.1-12.1 & 0.23 & 1549 & 460 & 0.31 & 112.8 & 1.5 & 0.0176 & 0.0002 & 56.54 & 1.3 & 0.0501 & 2.1 & 0.0481 & 3.0 \\
\hline ELM03-CH16.1-13.1 & -0.03 & 1925 & 710 & 0.38 & 115.6 & 1.5 & 0.0181 & 0.0002 & 55.27 & 1.3 & 0.0481 & 1.9 & 0.0469 & 2.3 \\
\hline ELM03-CH16.1-14.1 & 0.28 & 2735 & 1136 & 0.43 & 115.1 & 1.4 & 0.0180 & 0.0002 & 55.37 & 1.3 & 0.0505 & 1.6 & 0.0479 & 2.6 \\
\hline ELM03-CH16.1-15.1 & 0.09 & 1398 & 415 & 0.31 & 108.4 & 1.5 & 0.0170 & 0.0002 & 58.93 & 1.3 & 0.0489 & 2.3 & 0.0489 & 2.3 \\
\hline ELM03-CH16.1-16.1 & 0.20 & 2062 & 842 & 0.42 & 110.5 & 1.4 & 0.0173 & 0.0002 & 57.70 & 1.3 & 0.0498 & 1.8 & 0.0459 & 3.7 \\
\hline \multicolumn{15}{|c|}{ JT04-14, zircon: Bystinski pluton, south of Lupveem Batholith. Weighted mean average of ${ }^{207} \mathrm{~Pb}$-corrected ${ }^{206} \mathrm{~Pb} /{ }^{238} \mathrm{U}$ age $=116.9 \pm 2.5 \mathrm{Ma}, \mathrm{MSWD}=1.8$. } \\
\hline JT04-14-10 & 1.19 & 782 & 526 & 0.69 & 105.4 & 1.8 & 0.0165 & 0.0003 & 59.96 & 1.7 & 0.0576 & 2.5 & 0.0464 & 4.5 \\
\hline JT04-14-1C & 0.19 & 2611 & 579 & 0.23 & 212.2 & 3.4 & 0.0335 & 0.0006 & 29.83 & 1.6 & 0.0519 & 1.0 & 0.0511 & 1.3 \\
\hline JT04-14-2 & 0.08 & 7118 & 1506 & 0.22 & 120.5 & 2.0 & 0.0189 & 0.0003 & 52.97 & 1.6 & 0.049 & 0.8 & 0.0483 & 1.0 \\
\hline JT04-14-3C & 0.44 & 5644 & 1151 & 0.21 & 233.0 & 3.8 & 0.0368 & 0.0006 & 27.05 & 1.6 & 0.0543 & 0.6 & 0.0506 & 1.5 \\
\hline JT04-14-4 & 0.88 & 2975 & 1468 & 0.51 & 113.5 & 1.9 & 0.0178 & 0.0003 & 55.80 & 1.6 & 0.0553 & 1.2 & 0.0496 & 3.1 \\
\hline JT04-14-5 & 0.14 & 6461 & 3953 & 0.63 & 118.9 & 1.9 & 0.0186 & 0.0003 & 53.63 & 1.6 & 0.0495 & 0.9 & 0.0485 & 1.3 \\
\hline JT04-14-6 & 0.05 & 3410 & 385 & 0.12 & 114.9 & 1.9 & 0.0180 & 0.0003 & 55.55 & 1.6 & 0.0487 & 1.2 & 0.0479 & 1.5 \\
\hline JT04-14-7C & 0.19 & 3315 & 936 & 0.29 & 224.8 & 3.6 & 0.0355 & 0.0006 & 28.12 & 1.6 & 0.0522 & 0.9 & 0.0507 & 1.2 \\
\hline JT04-14-8 & 0.61 & 2292 & 565 & 0.25 & 132.9 & 2.2 & 0.0208 & 0.0003 & 47.72 & 1.7 & 0.0536 & 1.4 & 0.0499 & 2.8 \\
\hline JT04-14-9 & 0.05 & 2242 & 803 & 0.37 & 114.5 & 1.9 & 0.0179 & 0.0003 & 55.77 & 1.7 & 0.0487 & 1.4 & 0.0476 & 1.7 \\
\hline JT04-14-11 & 0.02 & 5907 & 2033 & 0.36 & 136.9 & 2.2 & 0.0215 & 0.0004 & 46.58 & 1.6 & 0.0490 & 0.7 & 0.0480 & 1.0 \\
\hline JT04-14-12C & 0.16 & 1403 & 559 & 0.41 & 214.4 & 3.5 & 0.0338 & 0.0006 & 29.53 & 1.7 & 0.0517 & 1.2 & 0.0502 & 1.7 \\
\hline JT04-14-13 & 1.52 & 7638 & 3846 & 0.52 & 119.8 & 2.0 & 0.0188 & 0.0003 & 52.50 & 1.6 & 0.0605 & 2.4 & 0.0481 & 5.1 \\
\hline JT04-14-14C & 0.86 & 1044 & 342 & 0.34 & 192.4 & 3.2 & 0.0303 & 0.0005 & 32.72 & 1.7 & 0.0568 & 1.4 & 0.0464 & 5.3 \\
\hline \multicolumn{15}{|c|}{ JT04-14, sphene: Bystinski pluton, south of Lupveem Batholith. Weighted mean average of ${ }^{207} \mathrm{~Pb}$-corrected ${ }^{206} \mathrm{~Pb} / 238 \mathrm{U}$ age $=106.4 \pm 2.5 \mathrm{Ma}, \mathrm{MSWD}=0.82$. } \\
\hline JT14-4.1 & 24.96 & 131 & 78 & 0.61 & 102.8 & 3.4 & 102.8 & 3.4 & 46.68 & 3.2 & 0.2463 & 2.1 & & \\
\hline JT14-10.1 & 19.39 & 172 & 39 & 0.24 & 103.6 & 3.4 & 103.6 & 3.4 & 49.74 & 3.2 & 0.2021 & 2.1 & 0.0548 & 59.8 \\
\hline JT14-9.1 & 17.41 & 226 & 121 & 0.55 & 104.2 & 3.3 & 104.2 & 3.3 & 50.70 & 3.1 & 0.1864 & 2.0 & 0.0833 & 23.0 \\
\hline JT14-2.2 & 17.63 & 218 & 36 & 0.17 & 104.4 & 3.3 & 104.4 & 3.3 & 50.47 & 3.1 & 0.1882 & 2.0 & 0.0476 & 49.5 \\
\hline JT14-12.1 & 25.35 & 136 & 82 & 0.62 & 106.2 & 3.6 & 106.2 & 3.6 & 44.96 & 3.2 & 0.2496 & 2.1 & 0.0419 & 86.9 \\
\hline JT14-17.1 & 18.07 & 220 & 115 & 0.54 & 106.6 & 3.4 & 106.6 & 3.4 & 49.12 & 3.1 & 0.1917 & 1.8 & & \\
\hline JT14-15.1 & 20.73 & 192 & 131 & 0.71 & 106.8 & 3.4 & 106.8 & 3.4 & 47.46 & 3.1 & 0.2129 & 2.0 & 0.0496 & 48.3 \\
\hline JT14-6.1 & 16.14 & 247 & 124 & 0.52 & 108.1 & 3.4 & 108.1 & 3.4 & 49.61 & 3.1 & 0.1764 & 1.9 & 0.0453 & 43.3 \\
\hline JT14-1.1 & 46.11 & 47 & 20 & 0.44 & 108.4 & 5.1 & 108.4 & 5.1 & 31.79 & 3.7 & 0.4147 & 2.6 & & \\
\hline JT14-11.1 & 23.68 & 161 & 80 & 0.51 & 108.7 & 3.6 & 108.7 & 3.6 & 44.90 & 3.2 & 0.2363 & 2.0 & 0.0721 & 36.5 \\
\hline JT14-19.1 & 16.17 & 235 & 160 & 0.70 & 109.0 & 3.4 & 109.0 & 3.4 & 49.17 & 3.1 & 0.1767 & 2.0 & & \\
\hline JT14-3.2 & 66.38 & 21 & 6 & 0.27 & 109.1 & 9.0 & 109.1 & 9.0 & 19.70 & 4.3 & 0.5763 & 2.8 & 0.3049 & 35.9 \\
\hline JT14-5.1 & 48.63 & 47 & 24 & 0.53 & 109.2 & 5.3 & 109.2 & 5.3 & 30.07 & 3.7 & 0.4349 & 2.5 & & \\
\hline JT14-8.1 & 26.97 & 133 & 96 & 0.75 & 109.4 & 3.7 & 109.4 & 3.7 & 42.66 & 3.2 & 0.2625 & 2.1 & & \\
\hline JT14-7.1 & 56.02 & 31 & 8 & 0.27 & 110.3 & 6.6 & 110.3 & 6.6 & 25.48 & 3.9 & 0.4938 & 2.7 & 0.2373 & 34.4 \\
\hline JT14-13.2 & 37.90 & 52 & 16 & 0.31 & 110.6 & 4.7 & 110.6 & 4.7 & 35.90 & 3.6 & 0.3495 & 2.8 & & \\
\hline JT14-16.1 & 32.12 & 132 & 78 & 0.61 & 111.5 & 3.8 & 111.5 & 3.8 & 38.90 & 3.2 & 0.3035 & 1.8 & & \\
\hline JT14-14.1 & 51.71 & 30 & 14 & 0.49 & 115.3 & 7.7 & 115.3 & 7.7 & 26.76 & 4.0 & 0.4596 & 4.2 & & \\
\hline JT14-2.1 & 50.91 & 36 & 10 & 0.27 & 115.8 & 6.1 & 115.8 & 6.1 & 27.09 & 3.8 & 0.4533 & 2.7 & 0.1249 & 80.8 \\
\hline
\end{tabular}


Table 2. Continued.

ELM03-CH12.1: Leucogranite dike. Weighted mean average of ${ }^{207} \mathrm{~Pb}$-corrected ${ }^{206} \mathrm{~Pb} /{ }^{238} \mathrm{U}$ age $=112.6 \pm 2.2 \mathrm{Ma}$ (MSWD=1.2).

\begin{tabular}{|c|c|c|c|c|c|c|c|c|c|c|c|c|c|c|}
\hline ELM03-CH12-1 & 0.47 & 996 & 411 & 0.43 & 111.5 & 1.9 & 0.0175 & 0.0003 & 57.03 & 1.7 & 0.0520 & 2.5 & 0.0467 & 4.9 \\
\hline ELM03-CH12-2 & 0.91 & 1652 & 798 & 0.50 & 115.1 & 1.9 & 0.0180 & 0.0003 & 54.99 & 1.7 & 0.0556 & 1.6 & 0.0444 & 6.5 \\
\hline ELM03-CH12-3 & 0.47 & 1298 & 410 & 0.33 & 115.3 & 1.9 & 0.0180 & 0.0003 & 55.16 & 1.7 & 0.0521 & 1.8 & 0.0486 & 3.4 \\
\hline ELM03-CH12-4 & 0.07 & 538 & 190 & 0.36 & 1070.4 & 17.3 & 0.1806 & 0.0032 & 5.53 & 1.7 & 0.0756 & 1.0 & 0.0746 & 1.2 \\
\hline ELM03-CH12-4.R & 0.42 & 1491 & 477 & 0.33 & 130.7 & 2.2 & 0.2780 & 0.0053 & 3.58 & 1.7 & 0.1008 & 0.8 & 0.0997 & 0.8 \\
\hline ELM03-CH12-5R & 0.93 & 1324 & 451 & 0.35 & 134.0 & 2.2 & 0.0210 & 0.0004 & 47.18 & 1.7 & 0.0561 & 1.6 & 0.0536 & 2.4 \\
\hline ELM03-CH12-6 & 0.14 & 1914 & 745 & 0.40 & 118.5 & 2.0 & 0.0186 & 0.0003 & 53.83 & 1.7 & 0.0495 & 1.6 & 0.0495 & 1.6 \\
\hline ELM03-CH12-6C & 0.05 & 486 & 23 & 0.05 & 717.0 & 11.9 & 0.1176 & 0.0021 & 8.50 & 1.7 & 0.0637 & 1.7 & 0.0634 & 1.7 \\
\hline ELM03-CH12-7 & 0.35 & 2007 & 689 & 0.35 & 119.4 & 2.0 & 0.0187 & 0.0003 & 53.31 & 1.7 & 0.0512 & 1.4 & 0.0481 & 2.8 \\
\hline ELM03-CH12-8 & 0.65 & 1686 & 606 & 0.37 & 113.0 & 1.9 & 0.0177 & 0.0003 & 56.21 & 1.7 & 0.0535 & 2.7 & 0.0477 & 4.6 \\
\hline ELM03-CH12.9 & 0.40 & 1228 & 580 & 0.49 & 112.5 & 1.9 & 0.0176 & 0.0003 & 56.56 & 1.7 & 0.0514 & 1.9 & 0.0495 & 3.3 \\
\hline ELM03-CH12.10 & 1.43 & 1439 & 589 & 0.42 & 109.4 & 1.8 & 0.0171 & 0.0003 & 57.61 & 1.7 & 0.0596 & 1.7 & 0.0503 & 5.2 \\
\hline ELM03-CH12.11 & 1.66 & 1853 & 854 & 0.48 & 111.4 & 1.9 & 0.0174 & 0.0003 & 56.42 & 1.7 & 0.0614 & 1.4 & 0.0493 & 5.7 \\
\hline ELM03-CH12-12 & 0.15 & 1929 & 923 & 0.49 & 114.2 & 1.9 & 0.0179 & 0.0003 & 55.85 & 1.7 & 0.0495 & 1.5 & 0.0471 & 2.6 \\
\hline ELM03-CH12-13 & 9.06 & 1860 & 3109 & 1.73 & 124.0 & 3.5 & 0.0194 & 0.0006 & 46.81 & 1.7 & 0.1206 & 11.7 & 0.0646 & 32.7 \\
\hline
\end{tabular}

JT04-46: Weighted mean average of ${ }^{207} \mathrm{~Pb}$-corrected ${ }^{206} \mathrm{~Pb} /{ }^{238} \mathrm{U}$ age $=114.7 \pm 1.8 \mathrm{Ma}$, MSWD=0.75.

\begin{tabular}{|c|c|c|c|c|c|c|c|c|c|c|c|c|c|}
\hline JT04-46-1 & 0.40 & 816 & 326 & 0.41 & 112.7 & 1.9 & 0.0176 & 0.0003 & 56.45 & 1.7 & 0.0515 & 2.1 & 0.0487 \\
\hline JT04-46-2 & 0.10 & 698 & 298 & 0.44 & 115.7 & 2.0 & 0.0181 & 0.0003 & 55.18 & 1.7 & 0.0491 & 2.3 & 0.0468 \\
\hline JT04-46-3 & 4.44 & 535 & 135 & 0.26 & 257.5 & 4.6 & 0.0407 & 0.0007 & 23.45 & 1.7 & 0.0870 & 1.3 & 0.0843 \\
\hline JT04-46-4 & 0.80 & 670 & 227 & 0.35 & 115.6 & 2.0 & 0.0181 & 0.0003 & 54.80 & 1.7 & 0.0547 & 2.3 & 0.0483 \\
\hline JT04-46-5 & 0.91 & 861 & 381 & 0.46 & 112.3 & 1.9 & 0.0176 & 0.0003 & 56.41 & 1.7 & 0.0555 & 2.1 & 0.0483 \\
\hline JT04-46-6 & 0.26 & 686 & 270 & 0.41 & 117.1 & 2.0 & 0.0183 & 0.0003 & 54.41 & 1.7 & 0.0504 & 2.5 & 0.0396 \\
\hline JT04-46-7 & 0.55 & 406 & 123 & 0.31 & 117.0 & 2.1 & 0.0183 & 0.0003 & 54.32 & 1.8 & 0.0527 & 3.3 & 0.0489 \\
\hline JT04-46-8 & 0.71 & 344 & 116 & 0.35 & 113.9 & 2.1 & 0.0178 & 0.0003 & 55.71 & 1.9 & 0.0539 & 3.5 & 0.0510 \\
\hline JT04-46-9 & 0.48 & 1287 & 807 & 0.65 & 115.1 & 1.9 & 0.0180 & 0.0003 & 55.24 & 1.7 & 0.0521 & 1.9 & 0.0475 \\
\hline JT04-46-10 & 0.64 & 597 & 196 & 0.34 & 104.3 & 1.9 & 0.0163 & 0.0003 & 60.94 & 1.8 & 0.0532 & 3.7 & 0.0492 \\
\hline JT04-46-11 & 1.19 & 470 & 159 & 0.35 & 113.6 & 2.1 & 0.0178 & 0.0003 & 55.56 & 1.8 & 0.0577 & 6.0 & 0.0527 \\
\hline
\end{tabular}

\begin{tabular}{|c|c|c|c|c|c|c|c|c|c|c|c|c|c|c|}
\hline \multicolumn{15}{|c|}{ JT04-4, zircon: Pyrkanai pluton. Weighted mean average of ${ }^{207} \mathrm{~Pb}$-corrected ${ }^{206} \mathrm{~Pb} /{ }^{238} \mathrm{U}$ age $=112.4 \pm 1.6 \mathrm{Ma}, \mathrm{MSWD}=0.032}$. \\
\hline JT04-4-1.1 & -0.15 & 1082 & 792 & 0.76 & 107.4 & 1.5 & 0.0168 & 0.0002 & 59.59 & 1.4 & 0.047 & 2.6 & 0.0460 & 3.0 \\
\hline JT04-4-2.1 & 0.93 & 1719 & 758 & 0.46 & 111.7 & 1.5 & 0.0175 & 0.0002 & 56.68 & 1.3 & 0.0556 & 2.7 & 0.0489 & 5.0 \\
\hline JT04-4-3.1 & 0.20 & 1386 & 449 & 0.33 & 111.3 & 1.5 & 0.0174 & 0.0002 & 57.31 & 1.3 & 0.0498 & 2.5 & 0.0463 & 4.5 \\
\hline JT04-4-4.1 & 0.58 & 1897 & 775 & 0.42 & 106.7 & 1.4 & 0.0167 & 0.0002 & 59.56 & 1.3 & 0.0527 & 2.0 & 0.0494 & 3.5 \\
\hline JT04-4-5.1 & 1.17 & 1703 & 701 & 0.43 & 107.5 & 1.5 & 0.0168 & 0.0002 & 58.77 & 1.3 & 0.0575 & 1.9 & 0.0494 & 5.0 \\
\hline JT04-4-6.1 & 0.45 & 1041 & 479 & 0.48 & 105.4 & 1.5 & 0.0165 & 0.0002 & 60.40 & 1.4 & 0.0517 & 2.6 & 0.0467 & 6.1 \\
\hline JT04-4-7.1 & -0.12 & 1319 & 407 & 0.32 & 111.3 & 1.5 & 0.0174 & 0.0002 & 57.48 & 1.3 & 0.0473 & 2.4 & 0.0473 & 2.4 \\
\hline JT04-4-8.1 & -0.16 & 2082 & 724 & 0.36 & 113.8 & 1.4 & 0.0178 & 0.0002 & 56.24 & 1.3 & 0.0470 & 1.8 & 0.0466 & 2.0 \\
\hline JT04-4-9.1 & 0.01 & 1498 & 488 & 0.34 & 111.1 & 1.5 & 0.0174 & 0.0002 & 57.55 & 1.3 & 0.0483 & 2.2 & 0.0461 & 2.9 \\
\hline JT04-4-10.1 & 0.40 & 563 & 343 & 0.63 & 113.3 & 1.8 & 0.0177 & 0.0003 & 56.16 & 1.5 & 0.0515 & 3.5 & 0.0515 & 3.5 \\
\hline JT04-4-11.1 & 0.07 & 1547 & 674 & 0.45 & 113.2 & 1.5 & 0.0177 & 0.0002 & 56.41 & 1.3 & 0.0489 & 2.1 & 0.0482 & 2.4 \\
\hline JT04-4-12.1 & 0.16 & 1472 & 562 & 0.39 & 113.9 & 1.5 & 0.0178 & 0.0002 & 56.01 & 1.3 & 0.0496 & 2.8 & 0.0496 & 2.8 \\
\hline JT04-4-13.1 & -0.11 & 1074 & 311 & 0.30 & 107.3 & 1.5 & 0.0168 & 0.0002 & 59.66 & 1.4 & 0.0473 & 2.7 & 0.0431 & 4.9 \\
\hline \multicolumn{15}{|c|}{ JT04-4, sphene: Pyrkanai pluton. Weighted mean average of ${ }^{207} \mathrm{~Pb}$-corrected ${ }^{206} \mathrm{~Pb} /{ }^{238} \mathrm{U}$ age $=110.6 \pm 1.7 \mathrm{Ma}$, MSWD=1.04. } \\
\hline & & & & & & & & & & & & & & \\
\hline JT4-2.1 & & 144 & 421 & & & 3.9 & & 0.0006 & & 3.3 & & 2.2 & & 82.9 \\
\hline JT4-3.1 & 24.72 & 129 & 461 & 3.68 & 108.1 & 3.8 & 0.0169 & 0.0006 & 44.50 & 3.3 & 0.2446 & 2.3 & 0.0446 & 80.9 \\
\hline JT4-4.1 & 21.05 & 376 & 269 & 0.74 & 114.7 & 3.8 & 0.0179 & 0.0006 & 43.99 & 3.3 & 0.2157 & 1.3 & 0.0995 & 38.1 \\
\hline JT4-5.1 & 23.67 & 233 & 638 & 2.83 & 101.5 & 3.2 & 0.0159 & 0.0005 & 48.10 & 3.1 & 0.2361 & 1.5 & 0.0400 & 57.2 \\
\hline JT4-6.1 & 41.86 & 171 & 171 & 1.04 & 105.7 & 3.7 & 0.0165 & 0.0006 & 35.16 & 3.1 & 0.3808 & 1.2 & 0.0612 & 53.5 \\
\hline JT4-7.1 & 22.42 & 281 & 249 & 0.92 & 107.9 & 3.4 & 0.0169 & 0.0005 & 45.98 & 3.0 & 0.2263 & 1.4 & 0.0401 & 48 \\
\hline JT4-7.2 & 31.84 & 249 & 203 & 0.84 & 113.8 & 3.7 & 0.0178 & 0.0006 & 38.28 & 3.1 & 0.3014 & 1.4 & & \\
\hline JT4-8.1 & 25.41 & 128 & 482 & 3.90 & 114.2 & 3.9 & 0.0179 & 0.0006 & 41.74 & 3.2 & 0.2503 & 2.1 & 0.1196 & 22.1 \\
\hline JT4-9.1 & 22.47 & 212 & 127 & 0.62 & 113.5 & 3.6 & 0.0178 & 0.0006 & 43.66 & 3.1 & 0.2269 & 1.7 & & \\
\hline JT4-10.1 & 24.29 & 181 & 209 & 1.20 & 109.5 & 3.6 & 0.0171 & 0.0006 & 44.19 & 3.2 & 0.2413 & 1.8 & 0.0577 & 50.5 \\
\hline JT4-11.1 & 24.06 & 109 & 348 & 3.30 & 110.9 & 4.1 & 0.0174 & 0.0006 & 43.76 & 3.5 & 0.2394 & 2.2 & 0.0489 & 73.4 \\
\hline JT4-11.2R & 26.07 & 125 & 278 & 2.29 & 112.5 & 3.9 & 0.0176 & 0.0006 & 42.00 & 3.3 & 0.2554 & 2.3 & 0.0993 & 32.5 \\
\hline JT4-12.1 & 22.32 & 237 & 182 & 0.79 & 111.5 & 3.5 & 0.0174 & 0.0006 & 44.54 & 3.1 & 0.2256 & 1.6 & & \\
\hline JT4-13.1 & 23.27 & 121 & 289 & 2.46 & 111.5 & 3.8 & 0.0174 & 0.0006 & 43.99 & 3.3 & 0.2332 & 2.4 & 0.0785 & 42.2 \\
\hline JT4-14.1 & 25.38 & 146 & 200 & 1.41 & 107.4 & 3.7 & & 0.0006 & & 3.2 & 0.2498 & 2.5 & 0.0695 & 43.4 \\
\hline JT4-15.1 & 24.12 & 129 & 287 & 2.30 & 117.2 & 4.0 & 0.0183 & 0.0006 & 41.37 & 3.3 & 0.2401 & 2.2 & 0.0677 & 45.4 \\
\hline JT4-16.1 & 25.20 & 123 & 379 & 3.19 & 110.8 & 3.8 & 0.0173 & 0.0006 & 43.16 & 3.3 & 0.2485 & 2.2 & 0.0805 & 53.4 \\
\hline JT4-17.1 & 23.55 & 170 & 229 & 1.39 & 111.3 & 3.6 & 0.0174 & 0.0006 & 43.92 & 3.2 & 0.2354 & 1.9 & & \\
\hline
\end{tabular}


Table 2. Continued.

\begin{tabular}{lllllllllllllllll} 
AN-100: Granite from South Anyui Suture Zone. Weighted mean average of ${ }^{207} \mathrm{~Pb}$-corrected ${ }^{206} \mathrm{~Pb} /{ }^{238} \mathrm{U}$ age $=108.5 \pm 2.7 \mathrm{Ma}, \mathrm{MSWD}=0.82$. \\
AN100.1 & 0.32 & 146 & 62 & 0.44 & 102.9 & 2.8 & 0.0161 & 0.0004 & 61.97 & 2.7 & 0.0506 & 6 & 0.0506 & 6 \\
AN100-2.1 & -0.56 & 271 & 120 & 0.46 & 110.6 & 2.7 & 0.0173 & 0.0004 & 58.09 & 2.5 & 0.0438 & 4.6 & 0.0438 & 4.6 \\
AN100-3.1 & 0.16 & 614 & 189 & 0.32 & 119.6 & 2.7 & 0.0187 & 0.0004 & 53.33 & 2.3 & 0.0496 & 2.6 & 0.0442 & 5.8 \\
AN100-4.1 & 0.02 & 335 & 167 & 0.52 & 108.8 & 2.7 & 0.017 & 0.0004 & 58.73 & 2.5 & 0.0484 & 3.9 & 0.0413 & 9.4 \\
AN100-5.1 & 0.59 & 376 & 193 & 0.53 & 108.4 & 2.6 & 0.017 & 0.0004 & 58.64 & 2.4 & 0.0528 & 3.6 & 0.0507 & 4.6 \\
AN100-6.1 & 0.17 & 296 & 111 & 0.39 & 109.2 & 2.7 & 0.0171 & 0.0004 & 58.42 & 2.4 & 0.0495 & 4.1 & 0.0495 & 4.1 \\
AN100-7.1 & 2.93 & 466 & 263 & 0.58 & 109.2 & 2.6 & 0.0171 & 0.0004 & 56.83 & 2.3 & 0.0714 & 2.8 & 0.0568 & 10.6 \\
AN100-8.1 & 0.13 & 415 & 255 & 0.63 & 109.7 & 2.6 & 0.0172 & 0.0004 & 58.18 & 2.4 & 0.0492 & 3.4 & 0.0474 & 4.4 \\
\hline
\end{tabular}

Analytical techniques

Zircon and sphene U-Pb analyses are conducted on the SHRIMP-RG (reverse geometry) ion microprobe co-operated by US Geological Survey and Stanford University in the SUMAC facility at Stanford University. Minerals, concentrated by standard heavy mineral separation processes and hand picked for final purity, are mounted on double stick tape on glass slides in $1 \times 6$ mm rows, cast in epoxy, ground and polished to a 1 micron finish on a $25 \mathrm{~mm}$ diameter by $4 \mathrm{~mm}$ thick disc. All grains are imaged with transmitted light and reflected light (and incident light if needed) on a petrographic microscope, and with cathodoluminescence(zircons) and back scattered electrons (sphene) as needed on a JEOL 5600 SEM to identify internal structure, inclusions and physical defects. The mounted grains are washed with EDTA solution and distilled water, dried in a vacuum oven, and coated with Au. Mounts typically sit in a loading chamber at high pressure (107 torr) for several hours before being moved into the source chamber of the SHRIMP-RG. Secondary ions are generated from the target spot with an O2-primary ion beam varying from 4-6nA. The primary ion beam typically produces a spot with a diameter of 20-40 microns and a depth of 1-2 microns for an analysis time of 9-12 min. Nine peaks are measured sequentially for zircons (the SHRIMP-RG is limited to a single collector, usually an EDP electron multiplier): 90Zr216O, 204Pb, Background (0.050 mass units above $204 \mathrm{~Pb}), 206 \mathrm{~Pb}, 207 \mathrm{~Pb}$, 208Pb, 238U, 248Th16O, 254U16O. 40Ca48 (Ti, Ca) 21604 is substituted for 90Zr2O in sphene. Autocentering on selected peaks and guide peaks for low or variable abundance peaks (i.e. $96 \mathrm{Zr} 216 \mathrm{O} 0.165$ mass unit below 204Pb) are used to improve the reliability of locating peak centers. The number of scans through the mass sequence and counting times on each peak are varied according to sample age and $U$ and Th concentrations to improve counting statistics and age precision. Measurements are made at mass resolutions of 6000-8000 (10\% peak height) which eliminates all interfering atomic species. The SHRIMP-RG was designed to provide higher mass resolution than the standard forward geometry of the SHRIMP I and II (Clement and Compston, 1994). This design also provides very clean backgrounds and combined with the high mass resolution, the acid washing of the mount, and rastering the primary beam for 90-120 s over the area to be analyzed before data is collected, assures that any counts found at mass of $204 \mathrm{~Pb}$ are actually $\mathrm{Pb}$ from the mineral and not surface contamination. In practice, greater than $95 \%$ of the spots analyzed have no common $\mathrm{Pb}$. Concentration data for zircons are standardized against zircon standard $\mathrm{CZ3}$ (550 ppm U) and age data against R33 (419 Ma, quartz diorite of Braintree complex, Vermont) which are analyzed repeatedly throughout the duration of the analytical session. Concentration and age data for sphenes are standardized against BLR-1 with an age of 1051 Ma and an average U concentration of $250 \mathrm{ppm}$. Data reduction follows the methods described by Williams (1997) and Ireland and Williams (2003) and uses the Squid and Isoplot programs of Ken Ludwig.

\subsubsection{Bystrinki pluton}

Along the southern extent of the Lupveem batholith, the large, equigranular, medium-grained, Bystrinski granitic pluton intrudes sillimanite-bearing amphibolite-facies country rocks (Fig. 5c). Sample JT04-14 was collected from this pluton at the contact with the metamorphic country rocks (Fig. 2, Table 1). Zircons from sample JT04-14 are subhedral and commonly embayed. Cathodoluminescence of these grains revealed complex internal zoning patterns, ubiquitous inclusions, and distinct zoned cores. Rims are unzoned, and typically dark in CL (commonly attributed to high $\mathrm{U}$ content). The U-Pb zircon data from this pluton are discordant and contain at least two age populations. A TeraWasserburg concordia diagram for the analyses of both zircon cores and rims from this sample is shown in Fig. 9. The analyses are discordant and likely reflect secondary $\mathrm{Pb}$ loss from these zircons. The cores of these grains are Early Jurassic to Triassic ${ }^{(207} \mathrm{Pb}$ corrected ${ }^{206} \mathrm{~Pb} /{ }^{238} \mathrm{U}$ ages range from 191.6-232.9 Ma; Table 2) and discordant. Six analyses of zircon rims produced a weighted mean age of $116.9 \pm 2.5 \mathrm{Ma}$ (MSWD=1.8). All of the data show some scatter above concordia indicative of $\mathrm{Pb}$ 'loss. The Bystrinki pluton also contains large $(>100 \mu \mathrm{m})$, euhedral, igneous sphene. U-Pb SHRIMP-RG analyses of 19 grains produced a linear array on a concordia diagram (Fig. 10), and a low MSWD of 0.82 which indicates a single age population. The lower intercept of $105.5 \pm 2.0 \mathrm{Ma}$ is within error of the weighted mean average age of $106.4 \pm 2.5 \mathrm{Ma}$. The upper intercept represents the isotopic composition of the common $\mathrm{Pb}$ component. This result is considerably younger than the age indicated by the zircon analyses, but is in very close agreement with the ${ }^{40} \mathrm{Ar} /{ }^{39} \mathrm{Ar}$ biotite ages from metamorphic country rocks as discussed below. Because country rocks adjacent to this pluton equilibrated at upper amphibolite grade with sillimanite stable, the sphene age of $106.4 \pm 2.5 \mathrm{Ma}$ is interpreted as the time the pluton finally cooled through $\sim 700^{\circ} \mathrm{C}$. Because this pluton is cross-cutting, undeformed and unmetamorphosed, its age provides a minimum age for the amphibolite-facies metamorphism and attendant deformation of country rocks 

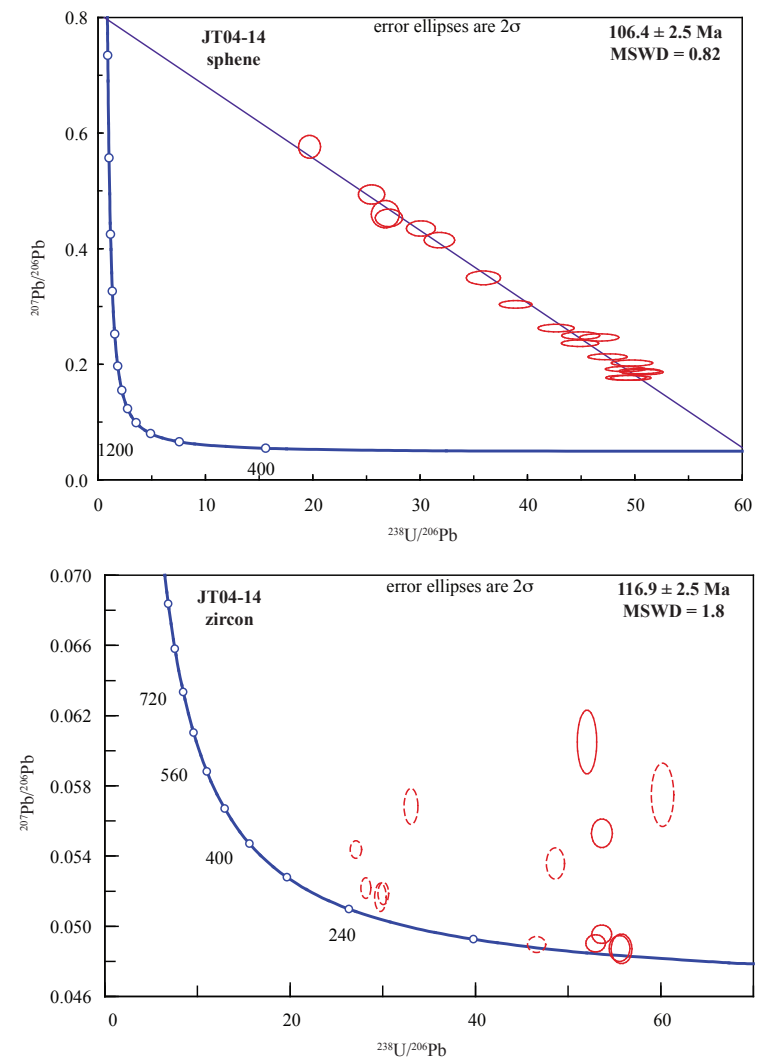

Fig. 9. Sample JT04-14: Bystrinki pluton of the Lupveem batholith collected near contact with country rock. Top: Tera-Wasserburg concordia diagram showing uncorrected U-Pb SHRIMP sphene ages. Final age assigned using the weighted mean ${ }^{207} \mathrm{~Pb}$-corrected ${ }^{206} \mathrm{~Pb} /{ }^{238} \mathrm{U}$ age of 22 analyses. Bottom: Tera-Wasserburg concordia diagram showing uncorrected U-Pb SHRIMP zircon ages. Final age assigned using the weighted mean ${ }^{207} \mathrm{~Pb}$-corrected ${ }^{206} \mathrm{~Pb} /{ }^{238} \mathrm{U}$ age of six rim analyses (solid ellipses).

during $D_{2}$. Two K-Ar ages from this pluton are $100 \pm 7$ and 127 $\pm 10 \mathrm{Ma}$ (Gel'man, 1963a, in Akinin and Kotlyar, 1997), and lie within error of the U-Pb ages.

\subsubsection{Leucocratic dikes and sills}

On the southernmost flank of the Alarmaut Massif, southeast of the Bystrinki pluton, numerous small irregular shaped bodies of leucogranite intrude country rocks. Here, S2 in the country rocks represents very high strains, with new biotite growth both parallel to the foliation and across it in some samples. The leucogranite body we sampled is itself weakly but visibly foliated, and is interpreted as possibly late or syndeformational with respect to $D_{2}$ (sample ELM03-CH12.1) (Fig. 2, Locality 3, Table 1). Zircons from sample ELM03CH12.1 vary in morphology from elongate to equant and from euhedral to subhedral. Cathodoluminescence images reveal complex zoning patterns and, in some grains, cores are clearly visible (Fig. 10). Sixteen analyses of this sam-
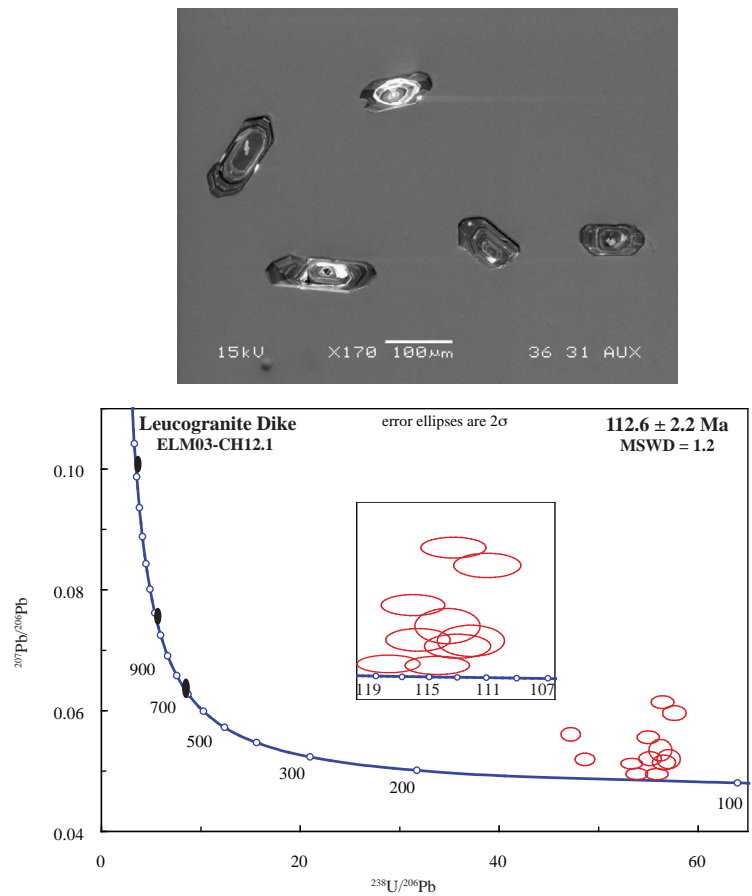

Fig. 10. Sample ELM03-CH12.1: leucogranite dike. Top: Cathodoluminescence image of representative zircons from this sample. Bottom: Tera-Wasserburg concordia diagram showing uncorrected U-Pb SHRIMP ages. Error ellipses are shown at two sigma uncertainty. Final age assigned using the weighted mean ${ }^{207} \mathrm{~Pb}$-corrected ${ }^{206} \mathrm{~Pb} /{ }^{238} \mathrm{U}$ age of nine analyses (shown in inset). Three concordant analyses of inherited cores are shown in filled ellipses.

ple, including three core/rim pairs, are shown in Fig. 10. The majority of the analyses are discordant, which may indicate secondary $\mathrm{Pb}$ loss or the incorporation of initial $\mathrm{Pb}$. The majority of the grains without cores, as well as the rims on grains with cores, yielded an Early Cretaceous age. Nine of the sixteen analyses (Fig. 10) gave a weighted mean age of $112.6 \pm 2.2 \mathrm{Ma}$ (MSWD=1.2), which is interpreted as the crystallization age. Analyses of the cores revealed Precambrian ages $\left({ }^{207} \mathrm{~Pb}\right.$ corrected ${ }^{206} \mathrm{~Pb} /{ }^{238} \mathrm{U}$ ages of $1581 \pm 27 \mathrm{Ma}$, $1070 \pm 17 \mathrm{Ma}$, and $717 \pm 12 \mathrm{Ma}$ ), and are interpreted to be inherited detrital zircons from the Devonian and older sedimentary country rocks or from the underlying basement.

\subsubsection{Kelilvun pluton}

Sample JT04-46 was collected from an isolated, circular pluton that consists of medium-grained, biotite-bearing homogeneous granite intrusive into Cretaceous sediments (Fig. 2, Locality 4, Table 1) Zircons from this sample are euhedral, but the grain-shapes range from nearly equant to needle-like (Fig. 11). Under CL, all grains show regular oscillatory zoning and no cores were visible. Eleven grains were analyzed. Nine out of the eleven analyses produced a weighted mean 
age of $114.7 \pm 1.8 \mathrm{Ma}$ (MSWD=0.75), which is interpreted as the crystallization age of this pluton (Fig. 11). One highly discordant Permian grain was discovered ${ }^{207} \mathrm{~Pb}$ corrected ${ }^{206} \mathrm{~Pb} /{ }^{238} \mathrm{U}$ age of $268 \pm 4.5 \mathrm{Ma}$ ) which might suggest inheritance of older material.

\subsubsection{Pyrkanai pluton}

Sample JT04-4 was collected from the Pyrkanai pluton, a large isolated, circular body of coarse-grained, porphyritic granite located north of Bilibino (Fig. 2, Locality 5 and Fig. 5e, Table 1). Zircons from sample JT04-4 are euhedral. Cathodoluminescence of these grains revealed very fine-scaled oscillatory zoning patterns and needle-like inclusions of apatite. No cores were observed inside the zircon grains, and only one population of grains was noted.

The uncorrected results from this sample are shown on Fig. 12. The weighted mean age of all thirteen analyses produced an age of $110.2 \pm 1.7$ (MSWD=3.4), which is interpreted as the crystallization age for these zircons. However, two age populations may be present in these zircons. Eight out of thirteen analyses produced a weighted mean age of $112.4 \pm 1.6 \mathrm{Ma}(\mathrm{MSWD}=0.66)$, while a subpopulation of five analyses produced slightly younger ages that are likely the result of $\mathrm{Pb}$ loss (Fig. 12). U-Pb analyses of sphene from the Pyrkanai pluton produce a weighted mean average age of $110.6 \pm 1.7 \mathrm{Ma}(\mathrm{MSWD}=1.04)$, which is consistent with the zircon age.

Previous K-Ar ages $(n=5)$ from this pluton have great scatter ranging from 84 to $130 \mathrm{Ma}$ (Akinin and Kotlyar, 1997). This range of ages is difficult to reconcile with the U-Pb data presented above, especially given the small size and compositional and textural homogeneity of this pluton.

\subsubsection{South Anyui pluton}

Sample AN-100 is a granite from the South Anyui suture zone that is post-tectonic with respect to penetrative deformation in the country rocks (Fig. 6). Zircons from the sample are typically euhedral, and show crude oscillatory zoning in CL (Fig. 13). Inclusions are common and were avoided during analysis. Eight analyses from this sample are shown in Fig. 13, and all but two are concordant within analytical error. Seven of the eight analyses were used to calculate a weighted mean age of $108.5 \pm 2.7 \mathrm{Ma}$, which is interpreted as the crystallization age for this pluton. One additional analysis yielded an age of $120 \pm 2.7 \mathrm{Ma}$, which may represent inheritance of older material (Fig. 13).

\subsection{Thermochronology}

\subsection{1 ${ }^{40} \mathrm{Ar} /{ }^{39} \mathrm{Ar}$ analyses}

${ }^{40} \mathrm{Ar} /{ }^{39} \mathrm{Ar}$ ages were obtained on four biotite separates from country rocks adjacent to the eastern side of the Lupveem batholith of the Alarmaut massif (Fig. 2, Locality a-d) to

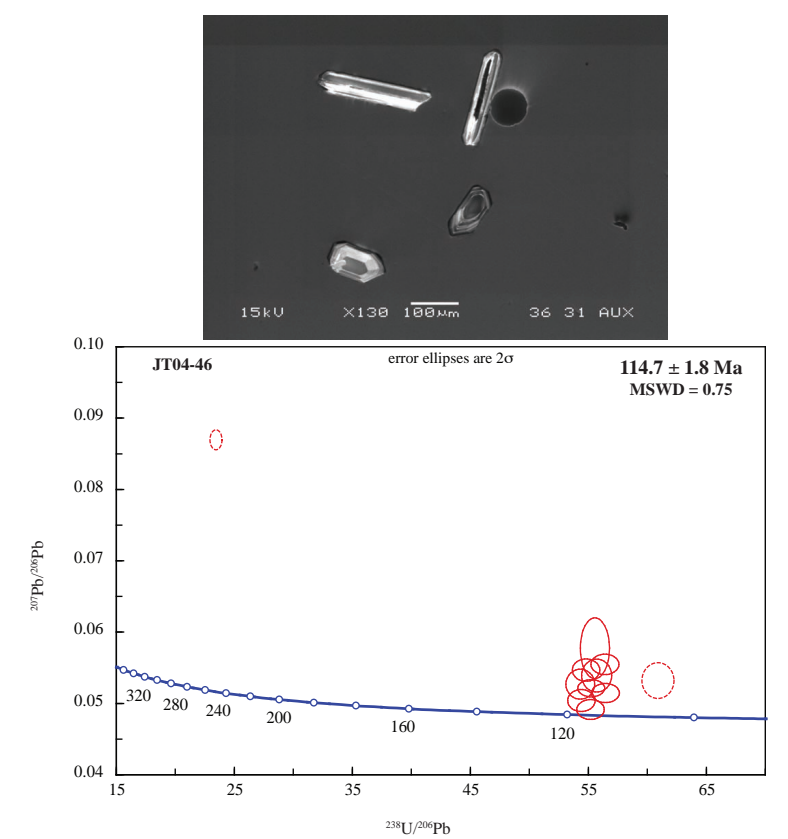

Fig. 11. Sample JT04-46: biotite-bearing granitic pluton located north of Bilibino. Top: Cathodoluminescence image of representative zircons from this sample. Bottom: Terra-Wasserburg concordia diagram showing uncorrected U-Pb SHRIMP ages. Error ellipses are shown at two sigma uncertainty. Final age assigned using the weighted mean ${ }^{207} \mathrm{~Pb}$-corrected ${ }^{206} \mathrm{~Pb} /{ }^{238} \mathrm{U}$ age of nine analyses (solid ellipses). Two additional analyses are shown (dashed ellipses).

better determine the minimum age for metamorphism and cooling from peak metamorphic conditions obtained during the second metamorphic and deformational event, $D_{2}$. Based on oriented thin section observations (e.g. Fig. 4d), we interpret the biotite in these samples to have grown syntectonically with the development of the $S_{2}$ foliation surfaces measured in the rocks. The resulting release spectra are shown in Fig. 14 and the data in Table 3. Three samples yielded ${ }^{40} \mathrm{Ar} /{ }^{39} \mathrm{Ar}$ plateau ages of $109.3 \pm 1.2,107.7 \pm 1.2$ and 103.0 $\pm 1.8 \mathrm{Ma}$ (Fig. 14). Sample ELM03 CH14.4, adjacent to the Lupveem batholith (Fig. 2) exhibits a climbing release spectrum with ages as old as $104 \mathrm{Ma}$ and a younger total fusion age of $90.7 \pm 1.2 \mathrm{Ma}$, a pattern compatible with slow cooling. The closure temperature of biotite to argon diffusion is about $300^{\circ} \mathrm{C}$ (Harrison et al., 1998). The ages obtained are slightly younger than the age of crystallization of the nearby plutons, and are close in age to the U-Pb age of sphene dated from the Bystrinki pluton (106.4 $\pm 2.5 \mathrm{Ma})$. Given the metamorphic grade of the rocks (upper greenschist to amphibolite, representing temperatures on the order of $450-600^{\circ} \mathrm{C}$ ) and the lower closure temperature of biotite, the ages obtained most likely represent cooling of the rocks from peak metamorphic conditions at depth after a protracted history of magmatism and high heat flow. 
Table 3. ${ }^{40} \mathrm{Ar} /{ }^{39} \mathrm{Ar}$ data.

\begin{tabular}{|c|c|c|c|c|c|c|c|c|c|c|}
\hline Step & $\begin{array}{l}\text { Temp } \\
\left({ }^{\circ} \mathrm{C}\right)\end{array}$ & ${ }^{40} \mathrm{Ar} /{ }^{39} \mathrm{Ar}$ & ${ }^{37} \mathrm{Ar} /{ }^{39} \mathrm{Ar}$ & $\begin{array}{l}{ }^{36} \mathrm{Ar} /{ }^{39} \mathrm{Ar} \\
\times 10-15 \mathrm{~mol}\end{array}$ & ${ }^{39} \mathrm{Ar}_{K}$ & $\begin{array}{l}\mathrm{K} / \mathrm{Ca} \\
(\%)\end{array}$ & $\begin{array}{l}{ }^{40} \mathrm{Ar}^{*} \\
(\%)\end{array}$ & $\begin{array}{l}{ }^{39} \mathrm{Ar}(\%) \\
(\mathrm{Ma})\end{array}$ & Age & $\pm 1 \mathrm{~s}$ \\
\hline \multicolumn{11}{|c|}{ Map locality A. Sample ELM03 CH 27.1 (biotite). Total fusion age $105.4 \pm 0.6 \mathrm{Ma}, J=0.0010297 \pm 0.6 \%$. } \\
\hline 1 & 800 & 66.12 & -0.0018 & 0.0265 & 6.7527 & -286.7717 & $88.1 \%$ & $5.2 \%$ & 103.32 & 1.06 \\
\hline 2 & 850 & 62.19 & 0.0113 & 0.0085 & 14.3424 & 46.0853 & $95.9 \%$ & $18.0 \%$ & 105.72 & 0.69 \\
\hline 3 & 900 & 61.72 & 0.0104 & 0.0039 & 15.2729 & 49.8075 & $98.1 \%$ & $29.7 \%$ & 107.29 & 0.90 \\
\hline 4 & 950 & 62.72 & 0.0091 & 0.0016 & 9.4109 & 57.3845 & $99.2 \%$ & $37.0 \%$ & 110.18 & 1.51 \\
\hline 5 & 1000 & 61.54 & 0.0140 & -0.0014 & 8.3591 & 37.2125 & $100.6 \%$ & $43.4 \%$ & 109.67 & 1.15 \\
\hline 6 & 1050 & 180.26 & -8.5219 & 0.2594 & -0.0217 & -0.0614 & $57.8 \%$ & $43.3 \%$ & 177.72 & 605.64 \\
\hline 7 & 1100 & 61.58 & 0.0175 & 0.0014 & 30.3213 & 29.7743 & $99.3 \%$ & $66.6 \%$ & 108.28 & 0.21 \\
\hline 8 & 1200 & 61.37 & 0.0251 & 0.0021 & 42.1342 & 20.7411 & $99.0 \%$ & $98.9 \%$ & 107.61 & 0.11 \\
\hline 9 & 1250 & 63.16 & 0.4754 & 0.0497 & 1.4949 & 1.0933 & $76.6 \%$ & $100.0 \%$ & 86.41 & 2.48 \\
\hline \multicolumn{11}{|c|}{ Map locality B. Sample ELM03 CH 14.4 (biotite). Total fusion age $90.7 \pm 0.4 \mathrm{Ma}, J=0.0010299 \pm 0.6 \%$. } \\
\hline 1 & 600 & 78.34 & 0.2114 & 0.1496 & 3.0091 & 2.4595 & $43.5 \%$ & $3.6 \%$ & 62.33 & 1.40 \\
\hline 2 & 700 & 80.87 & 0.2130 & 0.1986 & 2.6894 & 2.4412 & $27.4 \%$ & $6.9 \%$ & 40.73 & 1.63 \\
\hline 3 & 800 & 84.60 & 0.3881 & 0.1719 & 6.9051 & 1.3396 & $39.9 \%$ & $15.2 \%$ & 61.75 & 0.63 \\
\hline 4 & 850 & 79.50 & 0.0702 & 0.1095 & 7.2299 & 7.4037 & $59.3 \%$ & $23.9 \%$ & 85.49 & 0.56 \\
\hline 5 & 900 & 75.52 & 0.0631 & 0.0801 & 5.2507 & 8.2412 & $68.6 \%$ & $30.3 \%$ & 93.82 & 0.78 \\
\hline 6 & 950 & 87.21 & 0.0610 & 0.1273 & 6.2820 & 8.5276 & $56.8 \%$ & $37.9 \%$ & 89.82 & 0.68 \\
\hline 7 & 1000 & 83.41 & 0.0548 & 0.1095 & 6.7325 & 9.4920 & $61.2 \%$ & $46.0 \%$ & 92.42 & 0.65 \\
\hline 8 & 1050 & 82.82 & 0.0614 & 0.1011 & 10.9191 & 8.4625 & $63.9 \%$ & $59.2 \%$ & 95.74 & 0.42 \\
\hline 9 & 1100 & 72.01 & 0.0348 & 0.0564 & 15.0206 & 14.9216 & $76.8 \%$ & $77.3 \%$ & 99.94 & 0.31 \\
\hline 10 & 1125 & 65.55 & 0.0206 & 0.0289 & 10.7906 & 25.2689 & $86.9 \%$ & $90.3 \%$ & 102.87 & 0.43 \\
\hline 11 & 1150 & 60.38 & 0.0348 & 0.0089 & 7.2847 & 14.9397 & $95.6 \%$ & $99.1 \%$ & 104.17 & 0.63 \\
\hline
\end{tabular}

Map locality C. Sample ELM03 CH 14.1 (biotite) Total fusion age 108.2 $\pm 0.3 \mathrm{Ma}, J=0.0010297 \pm 0.6 \%$.

\begin{tabular}{|c|c|c|c|c|c|c|c|c|c|c|}
\hline 1 & 700 & 75.88 & 0.2236 & 0.1273 & 2.8747 & 2.3252 & $50.40 \%$ & $2.50 \%$ & 69.71 & 1.46 \\
\hline 2 & 800 & 62.31 & --0.4429 & -0.0627 & 0.1678 & -1.1745 & $129.70 \%$ & $2.70 \%$ & 144.11 & 22.52 \\
\hline 3 & 850 & 62.56 & 0.0153 & 0.0069 & 11.8046 & 33.9095 & $96.70 \%$ & $12.90 \%$ & 109.03 & 0.33 \\
\hline 4 & 900 & 60.9 & 0.0126 & 0.0011 & 12.2209 & 41.4179 & $99.40 \%$ & $23.60 \%$ & 109.1 & 0.33 \\
\hline 5 & 950 & 60.91 & 0.0205 & -0.0003 & 6.8162 & 25.3853 & $100.10 \%$ & $29.50 \%$ & 109.83 & 0.59 \\
\hline 6 & 1000 & 61.1 & 0.0169 & -0.0001 & 7.1529 & 30.7305 & $100.00 \%$ & $35.70 \%$ & 110.1 & 0.58 \\
\hline 7 & 1050 & 60.88 & 0.0128 & 0.0005 & 25.3183 & 40.5068 & $99.70 \%$ & $57.80 \%$ & 109.36 & 0.17 \\
\hline 8 & 1100 & 61.39 & 0.0055 & 0.0026 & 33.8828 & 93.699 & $98.70 \%$ & $87.30 \%$ & 109.2 & 0.14 \\
\hline 9 & 1125 & 60.65 & 0.019 & 0.0002 & 9.582 & 27.3247 & $99.90 \%$ & $95.70 \%$ & 109.14 & 0.47 \\
\hline 10 & 1150 & 59.82 & 0.0475 & -0.0031 & 3.8859 & 10.9421 & $101.50 \%$ & $99.10 \%$ & 109.39 & 1.18 \\
\hline 11 & 1175 & 46.2 & 0.3992 & -0.0253 & 0.6137 & 1.3023 & $116.10 \%$ & $99.60 \%$ & 97.09 & 7.72 \\
\hline 12 & 1200 & 16.59 & 1.3423 & -0.0844 & 0.1945 & 0.387 & $249.70 \%$ & $99.80 \%$ & 75.76 & 24.67 \\
\hline 13 & 1250 & 72.28 & 0.6243 & 0.0976 & 0.179 & 0.8325 & $60.00 \%$ & $99.90 \%$ & 79 & 28.89 \\
\hline 14 & 1300 & 97.33 & 0.9089 & 0.2065 & 0.097 & 0.5717 & $37.20 \%$ & $100.00 \%$ & 66.35 & 58.52 \\
\hline \multicolumn{11}{|c|}{ Map locality D. Sample ELM03 CH 12.1 (biotite). Total fusion age $102.4 \pm 0.9 \mathrm{Ma}, J=0.0010297 \pm 0.6 \%$. } \\
\hline 1 & 700 & 79.38 & 0.0397 & 0.0942 & 1.4278 & 13.0907 & $64.9 \%$ & $3.3 \%$ & 93.29 & 2.42 \\
\hline 2 & 800 & 61.08 & 0.0137 & 0.0133 & 4.8726 & 37.9449 & $93.5 \%$ & $14.7 \%$ & 103.14 & 0.68 \\
\hline 3 & 850 & 57.99 & 0.0110 & 0.0027 & 6.9535 & 47.4631 & $98.6 \%$ & $31.0 \%$ & 103.22 & 0.49 \\
\hline 4 & 900 & 57.86 & 0.0082 & 0.0035 & 4.7797 & 63.1218 & $98.2 \%$ & $42.2 \%$ & 102.57 & 0.74 \\
\hline 5 & 950 & 57.68 & 0.0209 & 0.0014 & 4.9391 & 24.8282 & $99.2 \%$ & $53.7 \%$ & 103.33 & 0.74 \\
\hline 6 & 1000 & 56.95 & 0.0075 & -0.0013 & 5.2509 & 69.0462 & $100.6 \%$ & $66.0 \%$ & 103.43 & 0.74 \\
\hline 7 & 1050 & 57.07 & 0.0087 & 0.0000 & 8.3332 & 59.5242 & $99.9 \%$ & $85.5 \%$ & 102.97 & 0.49 \\
\hline 8 & 1100 & 55.52 & 0.0152 & -0.0054 & 4.9869 & 34.1069 & $102.8 \%$ & $97.2 \%$ & 103.05 & 0.87 \\
\hline 9 & 1125 & 44.03 & 0.0507 & -0.0359 & 0.7505 & 10.2575 & $124.0 \%$ & $98.9 \%$ & 98.71 & 5.99 \\
\hline 10 & 1150 & 18.16 & 0.3409 & -0.1101 & 0.2598 & 1.5248 & $278.8 \%$ & $99.6 \%$ & 91.81 & 17.83 \\
\hline 11 & 1175 & -127.40 & 1.4668 & -0.5229 & 0.0703 & 0.3541 & $-21.2 \%$ & $99.7 \%$ & 49.89 & 78.34 \\
\hline 12 & 1200 & -319.66 & 1.0007 & -1.1060 & 0.0368 & 0.5192 & $-2.2 \%$ & $99.8 \%$ & 13.34 & 215.86 \\
\hline 13 & 1250 & -64.62 & 1.3125 & -0.3756 & 0.0452 & 0.3958 & $-71.6 \%$ & $99.9 \%$ & 84.36 & 122.73 \\
\hline 14 & 1300 & -72.19 & 1.2221 & -0.4372 & 0.0366 & 0.4251 & $-78.8 \%$ & $100.0 \%$ & 103.11 & 163.24 \\
\hline
\end{tabular}


Table 4. Fission track counting and age data.

\begin{tabular}{|c|c|c|c|c|c|c|c|c|c|}
\hline \multirow{2}{*}{$\begin{array}{l}\text { Sample } \\
\text { number }\end{array}$} & \multirow{2}{*}{$\begin{array}{l}\text { No } \\
\text { Xls }\end{array}$} & \multicolumn{2}{|c|}{ Spontaneous } & \multicolumn{2}{|c|}{ Induced } & \multirow{2}{*}{$\begin{array}{l}P\left(\chi^{2}\right) \\
(\%)\end{array}$} & \multicolumn{2}{|c|}{ Dosimeter } & \multirow{2}{*}{$\begin{array}{l}\text { Age } \pm 1 \sigma \\
(\mathrm{Ma})\end{array}$} \\
\hline & & Rho-S & NS & Rho-I & NI & & Rho-D & ND & \\
\hline ELM03 CH 16.1 & 6 & 0.8754 & 177 & 2.4333 & 492 & 10 & 1.2154 & 3608 & $90 \pm 11$ \\
\hline
\end{tabular}

Note: Abbreviations are: No Xls, number of individual crystals (grains) dated; Rho-S, spontaneous track density $\left(\times 10^{6}\right.$ tracks per square centimeter); NS, number of spontaneous tracks counted; Rho-I, induced track density in muscovite external detector $\left(\times 10^{6}\right.$ tracks per square centimeter); NI, number of induced tracks counted; $P\left(\chi^{2}\right), \chi^{2}$ probability (Green, 1981); Rho-D, induced track density in external detector adjacent to dosimetry glass $\left(\times 10^{6}\right.$ tracks per square centimeter); ND, number of tracks counted in determining Rho-D. Age is the sample central fission track age (Galbraith and Laslett, 1993), calculated using zeta calibration method (Hurford and Green, 1983). Analyst: T. A. Dumitru.

Laboratory procedures were identical to those in Table DR2 of Unruh et al. (2007). Age calculations were done with program by J. P. Colgan. The quality of apatite in this sample was poor, primarily due to abundant dislocations and/or fluid inclusions in almost all grains. Therefore, inaccuracies in the age may somewhat exceed the one sigma uncertainty quoted above.
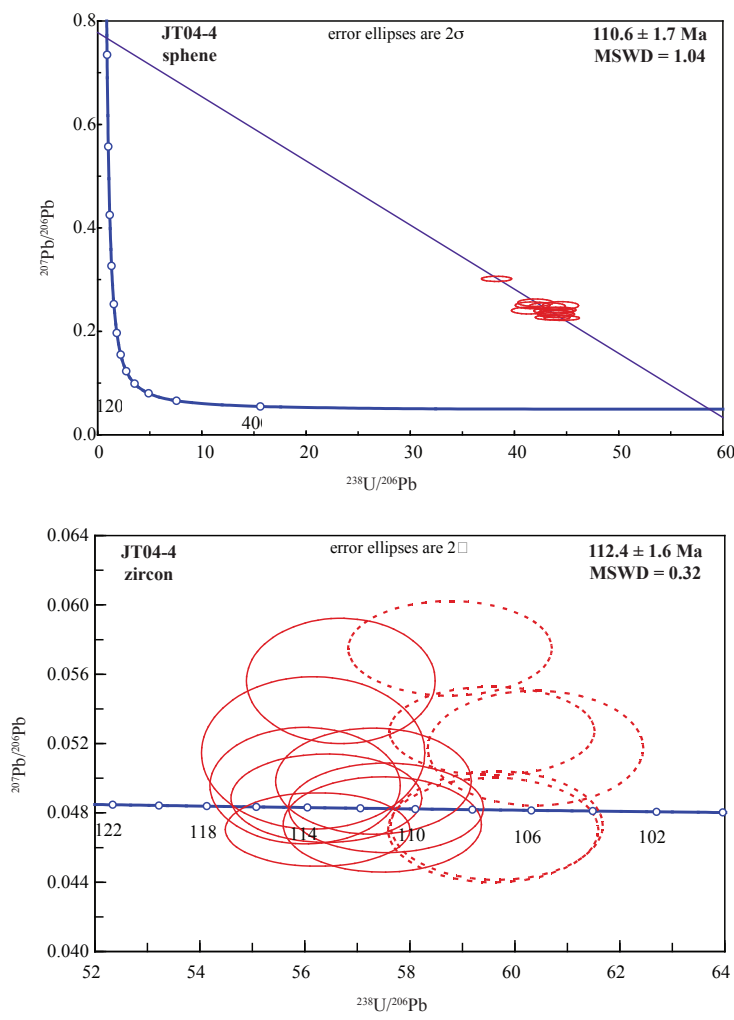

Fig. 12. Sample JT04-4: Pyrkanai pluton. Top: Terra-Wasserburg concordia diagram showing uncorrected U-Pb SHRIMP sphene ages. Error ellipses are shown at two sigma uncertainty. Final age assigned using the weighted mean ${ }^{207} \mathrm{~Pb}$-corrected ${ }^{206} \mathrm{~Pb} /{ }^{238} \mathrm{U}$ age of 19 analyses. Bottom: Terra-Wasserburg concordia diagram showing uncorrected U-Pb SHRIMP zircon ages. Error ellipses are shown at two sigma uncertainty. Final age assigned using the weighted mean ${ }^{207} \mathrm{~Pb}$-corrected ${ }^{206} \mathrm{~Pb} /{ }^{238} \mathrm{U}$ age of eight analyses (solid ellipses). Five additional data points (dashed ellipses) have likely experienced $\mathrm{Pb}$ loss.

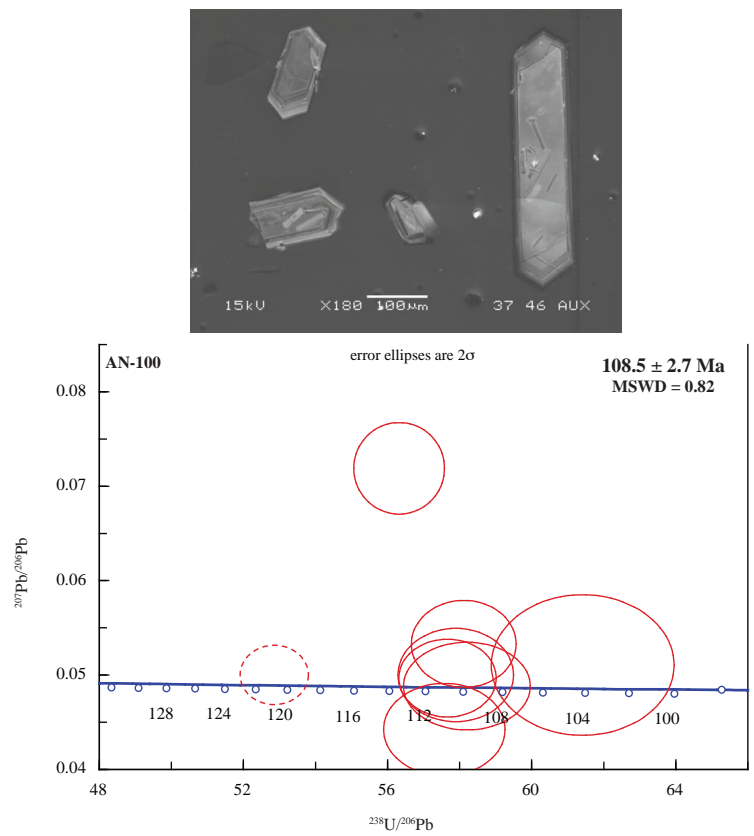

Fig. 13. Sample AN-100: granite from the South Anyui suture zone. Top: Cathodoluminescence image of representative zircons from this sample. Bottom: Terra-Wasserburg concordia diagram showing uncorrected U-Pb SHRIMP ages. Error ellipses are shown at two sigma uncertainty. Final age assigned using the weighted mean ${ }^{207} \mathrm{~Pb}$-corrected ${ }^{206} \mathrm{~Pb} /{ }^{238} \mathrm{U}$ age of all nine analyses (solid ellipses). One additional data point is shown (dashed ellipse).

\subsubsection{Fission track analyses}

Apatite fission track ages provide valuable information on the time and rate of low temperature cooling of rocks through the $120-80^{\circ} \mathrm{C}$ temperature range. Apatite was separated from 15 samples of igneous, metamorphic and sedimentary rocks from a transect extending east-northeast from the Alarmaut massif to the Arctic coastal plain along line of 

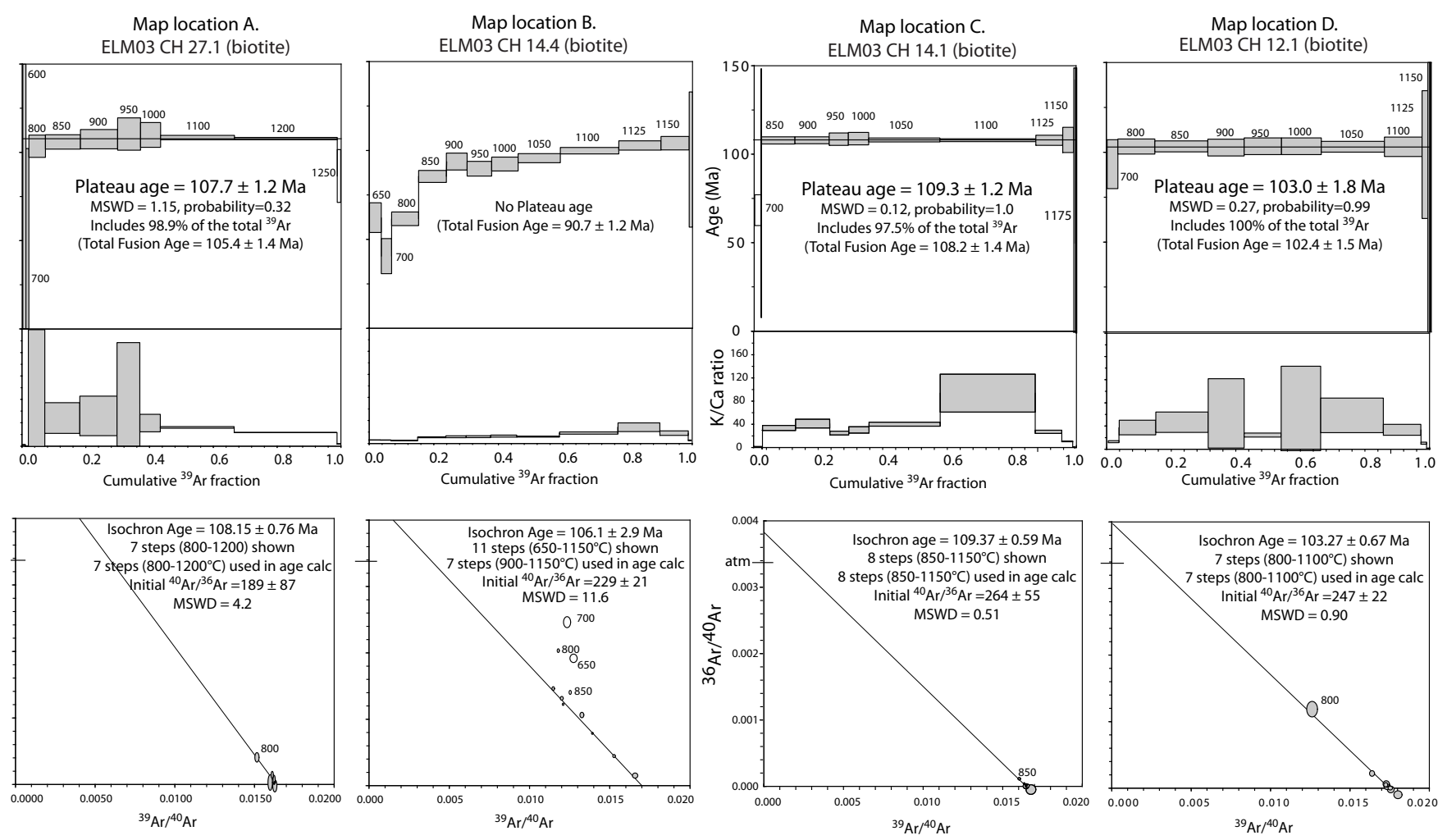

Fig. 14. ${ }^{40} \mathrm{Ar} /{ }^{39} \mathrm{Ar}$ release spectra for metamorphic biotite from samples collected adjacent to the Alarmaut massif; data is shown in Table 3 . All rectangular boxes and ellipses in plots are show with two sigma errors; values shown by open rectangles and ellipses are not used for calculations, ellipses on reverse isochron diagrams are blank-corrected, which cause them to plot below zero. Atm is atmospheric ${ }^{36} \mathrm{Ar} /{ }^{40} \mathrm{Ar}$ ratio.

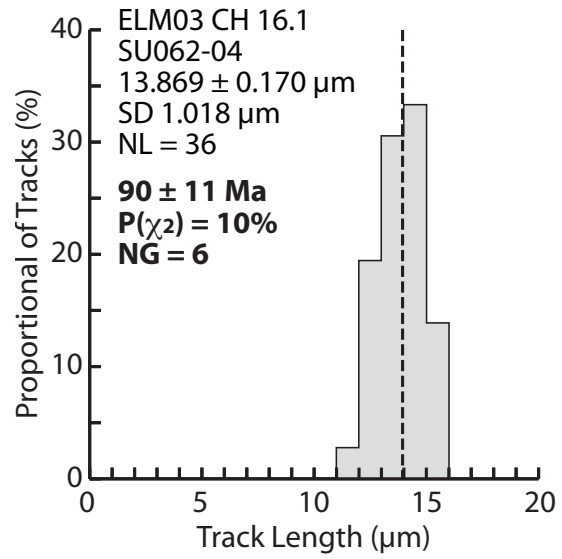

Fig. 15. Fission track age and confined track length measurements for sample ELM03 CH16.2, based on data shown in Table 4.

Sect. A-A'l'! (Fig. 2) to determine the cooling history of this gently-tilted crustal section (Fig. 2). None of the apatites were suitable for fission track dating due to a large number of fluid inclusions and dislocations in all grains. This discovery is consistent with evidence for extensive and ubiquitous hydrothermal alteration of country rocks during
Cretaceous magmatism. Only one granitic sample ELM03 $\mathrm{CH} 16.1$ (with a U-Pb age of $112 \mathrm{Ma}$ ) out of 15 samples yielded sufficient grains that could be analyzed and yielded an age of $90 \pm 11 \mathrm{Ma}$ (Fig. 15 and Table 4). Track length measurements are fairly unimodal with a mean of $13.869 \pm 0.170$ microns, indicating residence of the granite at temperatures above $120^{\circ} \mathrm{C}$ for a prolonged period after intrusion with relatively rapid passage through the $80-120^{\circ}$ temperature interval at $90 \pm 11 \mathrm{Ma}$. Despite its large error, this age is compatible with regional timing constraints for the formation of the overlying unconformity during the time interval 108 and $87 \mathrm{Ma}$ (age brackets provided by the youngest dated pluton beneath the unconformity and the oldest volcanic rocks erupted across the unconformity).

\section{Discussion and conclusions}

The age of the crystallization of zircons from the granitoid rocks of the Lupveem batholith and Bystrinski, Kelilvun and Pyrkanai plutons as well as a small pluton from the South Anyui suture zone were determined by the U-Pb method utilizing the USGS-Stanford University SHRIMP-RG and yielded ages ranging from $116.9 \pm 2.5 \mathrm{Ma}$ 
to $108.5 \pm 2.7 \mathrm{Ma}$. U-Pb sphene ages from the Bystrinki pluton, intruded into upper amphibolite facies rocks is significantly younger than its zircon age, $105.5 \pm 2.0 \mathrm{Ma}$ (sphene) as compared to $116.9 \pm 2.5 \mathrm{Ma}$ (zircon), indicating prolongued heating and/or slow cooling of these deeper levels of the crust. In contrast, the Pyrkanai pluton which yields concordant zircon $(110.2 \pm 1.7 \mathrm{Ma})$ and sphene $(110.6 \pm 1.7 \mathrm{Ma})$ ages is inferred to have been intruded into shallower levels of the crust as evidenced by its narrow andalusite-bearing contact aureole. All of the granites, based on clear crosscutting relations, post-date the formation of structures in the Anyui-Chukotka foldbelt. This regional deformational event is believed to be related to the collision of the Chukotka microcontinent with the Eurasian margin of NE Russia. Inception of deformation is best dated by the flood of synorogenic clastic strata derived from the south beginning in the latest Jurassic and continuing into the Early Cretaceous (Bondarenko et al., 2003; Baranov, 1996; Miller et al., 2009; Miller and Verzhbitski (this volume). In the SAZ, sediments as young as Hauterivian-Barremian $(136.4 \pm 2.0$ to 125.0 1.0 ; Gradstein et al., 2004) are involved in deformation. The oldest post-tectonic plutons we have dated are $116.9 \pm 2.5$, thus regional shortening-related deformation was over by this time. Penetrative deformation in the SAZ is cut by a granitic pluton dated as $108.5 \pm 2.7$ but our data do not rule out the possibility that brittle faulting could have occurred elsewhere in the SAZ after its intrusion.

The data presented here, obtained with the use of modern geochronologic methods, adds support to the timing of collision based on stratigraphic relations as discussed by Bondarenko and Luchitskaya (2003), Parfenov (1984), Seslavinskiy (1979), Sokolov et al. (2002) and Natal'in et al. (1999).

The close relationship between the intrusion of the Lupveem batholith and country rock metamorphism and deformation in the Alarmaut massif region and the orientation of dikes from plutons across this region suggest that the plutons dated were intruded during a second deformational event that at least locally, and possibly more widely at depth, produced ductile metamorphic fabrics that overprint regional shortening-related structures. The high-strain, sub-horizontal foliations formed during $D_{2}$ are related to vertical thinning of the crust and NE-SW to NW-SE subhorizontal stretching. Thus, widespread magmatism heralded a change in tectonic regime from regional shortening to extensional or transtensional at some time before about 116.9 $\pm 2.5 \mathrm{Ma}$. This extensional tectonic regime lasted from at least $\sim 120$ to $\sim 105 \mathrm{Ma}$, spanning the age (with associated errors) of the plutons dated. It represents an important tectonic event across Chukotka that has not been previously recognized or interpreted as extensional. Filatova and Khain (2007) for example, relate granite-cored uplifts in Chukotka like the Alarmaut massif to collision, and the subhorizontal fabrics in the Alarmaut massif to "nappe tectonics". At the more regional scale, E-W to NE-SW extension is well documented in the Pevek region north of Bilibino
(Miller and Verzhbitsky, this volume) where the relative and absolute timing of events is the same as suggested by this study.

${ }^{40} \mathrm{Ar} /{ }^{39} \mathrm{Ar}$ ages of biotite from metamorphic country rocks from structurally deepest exposures adjacent to the Lupveem batholith of the Alarmaut massif indicate that country rocks cooled to below $300^{\circ} \mathrm{C}$ together with the granites between 109-103 Ma. Based on apatite fission track dating, by about $90 \pm 11 \mathrm{Ma}$, erosion had beveled plutons and country rocks to a low-relief peneplain. The eruption of the oldest OCVB lavas across this erosional surface represents a southward step in magmatic activity to the Pacific margin of NE Russia.

Farther east in Chukotka, Cretaceous extension is well documented in the Bering Strait region of Alaska and Russia (e.g. Amato and Miller, 2004; recent summary and compilation of references in Akinin et al., 2009). However, here extension is at right angles to that documented in the Pevek and Bilibino region of Russia and Alaska, and is oriented approximately N-S. Based on existing geochronology, it appears that the age of plutonism and extension in the broader Bilibino-Pevek region may be slightly older in age than that in the Bering Strait region. Gneiss dome development and sub-horizontal fabrics in the Bering Strait region deform plutons as young as $105-103 \mathrm{Ma}$ and the gneiss domes themselves are inferred to have formed at about 90-95 Ma. At this time near Bilibino and Pevek, magmatism was largely over and the landscape deeply beveled by erosion. These changing relations with respect to the age of magmatism, extensional deformation as well as the direction of extension across Arctic Russia may be significant in terms of understanding the continental manifestations of the rift history of the Amerasian Basin of the Arctic Ocean. However, we are still very far from having the data needed to address where, how and why these changing relations take place. As discussed by Miller and Verzbitsky (this volume), we believe that magmatism and extension in Chukotka may represent the onshore consequence of early rifting in the Amerasian Basin, the timing and direction of which could be different from that generating the Canada Basin which lies further to the east (Fig. 2).

Acknowledgements. This study was carried out with gift funds in support of the Structural Geology and Tectonics Laboratory at Stanford (Elizabeth Miller, P. I.). Katkov acknowledges the financial help of the RUSSIAN BASIC RESEARCH FUND (projects 05-05 65052 and 06-05-64824), FTSNTP (leading scientific schools OF NSh-9664.2006.5). Akinin's visit to the US was partially funded by the School of Earth Sciences Blaustein visiting scholar funds. Katkov expresses his thanks to his thesis advisor S. D. Sokolov and to his friends and colleagues at the Laboratory of Structural Geology and Tectonics during his stay at Stanford and to USGS scientists Joe Wooden, Frank Mazdab and Brad Ito for their help using the SHRIMP-RG. A. Strickland acknowledges support of her work by Stanford University graduate fellowships, the USGS-Stanford SHRIMP-RG Lab and her parents. Final data compilation and 
preparation of this paper acknowledges the help of undergraduate student Julie Padilla supported by an American Chemical SocietyPRF Award 45432-AC8 to Elizabeth Miller. We thank the director and the geologists of the Bilibino Geological Survey who helped us with logistics and who shared their geological knowledge of the region with us. Finally, our work would not have been possible without the hard work of Marianna Tuchkova (GIN-RAS, Moscow) who organized our logistics and trips to Chukotka and the dedication and support of our track vehicle driver, mechanic and superb gourmet cook, Valodya Zhdankin. Very helpful reviews by L. Lane and A. Till are much appreciated.

\section{References}

Akinin, V. V., Hourigan, J. K., Wright, J. E., Miller, E. L., and Mishin, L. F.: New data on age of Okhotsk-Chukotka volcanic belt (U-Pb SHRIMP dating): Isotopic dating of processes of ore formation, magmatism, sedimentation, and metamorphism. Proceedings of 3rd all Russian conference on isotopic geochronology, Moscow, GEOS, 1, 22-26, 2006 (in Russian).

Akinin, V. V. and Khanchuk, A. I.: The Okhotsk-Chukotka Volcanogenic Belt: Age Revision Based on New 40Ar/39Ar and UPb Isotope Data, Dokl. Earth Sci., 405(8), 1131-1135, 2005.

Akinin, V. V. and Kotlyar, I. N.: GEOCHRON: The computer database of isotopic dating of minerals, rocks, and ores in Northeastern Siberia, in: Magmatism and mineralization in northeastern Siberia, edited by:Byalobzeski, S. G., Northeast Interdisciplinary Science Research Institute, Magadan, Russia, 314-318, 1997 (in Russian).

Akinin, V. V, Miller, E. L., and Wooden, J.: Petrology and Geochronology of crustal xenoliths from the Bering Strait region: Linking deep and shallow processes in extending continental crust, in: Crustal cross-secctions from the western North American Cordillera and elsewhere: Implications for tectonic and petrologic processes, edited by: Miller, R. B. and Snoke, A. W., Geol. Soc. Am. Special Paper, 4XX, in press, 2009.

Akimenko, G. I.: Crustal Cross Sections from the Western North American Cordillera and Elsewhere, in: Implications for Tectonic and Petrologic Processes, edited by: Miller, R. B. and Snoke, A. W., SPE456: ISBN 978-0-8137-2456-0, Geologic Map, R-58XXXV, XXXVI (Bilibino), 1:200000 scale, 2000.

Amato, J. M. and Miller, E. L.: Geologic map and summary of the evolution of the Kigluaik Mountains gneiss dome, Seward Peninsula, Alaska, in: Gneiss Domes in Orogeny, edited by: Whitney, D. L, Teyssier, C., and Siddoway, C. S., Geol. Soc. Am. Special Paper, 380, 295-306, 2004.

Baranov, M. A.: Nappe tectonics of the Myrgovaam Basin in North-western Central Chukotka, Tikhookean. Geol., 12, 441448, 1996.

Belik, G. Ya. and Sosunov, G. M. (Eds.): Geologic map of USSR: North-Eastern Geological Directorate, Anyuisko-Chaunskaya series, R-58-XXIX, XXX, 1 sheet, scale 1:200 000, 1969.

Bogdanov, N. A. and Til'man, S. M.: Tectonics and geodynamics of the northeast of Asia (explanatory note to the tectonic map), 54 pp., 1992.

Bondarenko, G. E. and Luchitskaya, M. T.: Tectonic evolution of the Alarmaut uplift, western Chukchi Peninsula, Northeastern Asia, Bull. MOIP, Geological Section, 78, 25-37, 2003.
Bondarenko, G. E., Soloviev, A. V., Tuchkova, M. I., Garver, J. I., and Podgornyi, I. I.: Age of detrital zircons from sandstones of the Mesozoic flysch formation in the South Anyui suture zone (western Chukoktka), Lithol. Miner. Resour., 38(2), 162-176, 2003.

Chasovitin, M. D. and Shpetnyi, A. P.: Geologic map of USSR: North-Eastern Geological Directorate, Anyuisko-Chaunskaya series, Map R-58-XXXIII, XXXIV, scale 1:200 000, 1964.

Cherniak, D. J.: Lead diffusion in titanite and preliminary results on the effects of radiation damage on $\mathrm{Pb}$ transport, Chem. Geol., 110, 177-194, 1993.

Clement, S. W. J. and Compston, W.: Ion probe parameters for very high resolution without loss of sensitivity, US Geological Survey Circular, 1107, 62 pp., 1994.

Dahl, P. S.: A crystal-chemical basis for $\mathrm{Pb}$ retention and fission track annealing systematics in U-bearing minerals, with implications for geochronology, Earth Planet. Sc. Lett., 150(3-4), 277290, 1997.

Dovgal, Yu. M.: National Geologic map of USSR, MinGeo Russ., Magadan, Anyui-Chaun Series, Sheet Q-58-IX, X, scale 1:200 000, 1964.

Filatova, N. I. and Khain, V. E.: Tectonics of the eastern Arctic region, Geotektonica, 3, 3-29, 2007.

Frost, B. R., Chamberlain, K. R., and Schumacher, J. C.: Sphene (titanite): phase relations and role as a geochronometer, Chemical Geology, 172, 131-148, 2000.

Galbraith, R. F. and Laslett, G. M: Statistical models for mixed fission track ages, Nucl. Tracks Rad. Meas., 21, 459-470, 1993.

Gel'man, M. L.: Triasovaya diabasovaya formatisiya Anyuiskoi zony (Chukotka) (Triassic diabase association in the Anuy fold zone (Chukotka), Geol. Geofiz., 2, 127-134, 1963 a.

Gel'man, M. L.: Deep facies of Cretaceous granites in the Anyui Zone, I. Reports on the Geology and Ores of the Northeastern USSR, SVGU, Magadan, 16, 213-233, 1963b.

Gel'man, M. L. (Ed.) and Sadovskii, A. I. : Geologic map of USSR: North-Eastern Geological Directorate, Anyuisko-Chaunskaya series, R-58-XXVII, XXVIII, 1 sheet, scale 1:200 000, 1967.

Gradstein, F., Ogg, J., and Smith, A.: A Geologic Time Scale, Cambridge University Press, 589 pp., 2004.

Grantz, A., May, S. D., and Hart, P. E.: Geology of Arctic continental margin of Alaska, in: The Geology of North America, v. L, The Arctic Ocean region, edited by: Grantz, A., Johnson, L., and Sweeney, J. F., Geol. Soc. Am., Boulder, CO, 257-288, 1990.

Grantz, A., May, S. D., Taylor, P. T., and Lawver, L. A.: Canada Basin, in: The Geology of North America, v. L, The Arctic Ocean region, edited by: Grantz, A., Johnson, L., and Sweeney, J. F., Geological Society of America, Boulder, CO, 397-402, 1990.

Green, P. F.: A new look at statistics in fission-track dating, Nucl. Tracks Rad. Meas., 5, 77-86, 1981.

Harrison, T. M., Duncan, I., and McDougall, I.: Diffusion of 40Ar in biotite: Temperature, pressure and compositional effects, Geochim. Cosmochim. Ac., 49, 2461-2468, 1985.

Hourigan, J. K. and Akinin, V. V.: Tectonic and chronostratigraphic implications of new 40Ar/39Ar geochronology and geochemistry of the Arman and Maltan-Ola volcanic fields, OkhotskChukotka volcanic belt, northeastern Russia, Geological Society of America Bulletin, 116, 637-654, 2004.

Hurford, A. J. and Green, P. F.: The zeta age calibration of fission- 
track dating, Chem. Geol., 41, 285-317, 1983.

Ireland, T. R. and Williams, I. S.: Considerations in zircon geochronology by SIMS, in: Zircon, edited by: Hanchar, J. M. and Hoskins, P. W. O., Rev. Mineral. Geochem., 53, 215-241, 2003.

Katkov, S. M., Miller, E. L., Podgorny, I. I., and Toro, J.: Deformation history of central Chukotka (Alarmaut Uplift), northeastern Arctic Russia, in: Origin of Northeastern Russia: Paleomagnetism, Geology and Tectonics, edited by: Stone, D. B., Geophys. Inst. Rept. UAG-R-330, University of Alaska, Fairbanks, AK, CD, 2006.

Kos'ko, M. K., Cecile, M. P., Harrison, J. C., Ganelin, V. G., Khandoshko, N. V., and Lopatin, B. G.: Geology of Wrangel Island, between Chukchi and East Siberian seas, northeastern Russia, Geological Survey of Canada, Bulletin, 461, 107 pp., 1993.

Lane, L. S, Layer, P. W, Cecile, M. P., and Kos'ko, M.: Ar-Ar ages from the Okhotsk-Chukotks magmatic arc, III Int. Conf. Arctic Margins (ICAM), Celle, Germany 12-16, 106-107, 1998.

Lawver, L. A., Grantz, A., and Gahagan, L.: Plate Kinematic evolution of the present Arctic region since the Ordovician, in: Tectonic Evolution of the Bering Shelf-Chukchi Sea-Arctic Margin and Adjacent Landmasses, edited by: Miller, E. L., Grantz, A., and Klemperer, S. L., Geol. Soc. Am. Special Paper, 360, 333358, 2002.

Ludwig, K. R.: Isoplot 3.00, a geochronological toolkit for Excel, Berkeley Geochron. Center Spec. Pub. 4., 2003.

Mezger, K. and Krogstad, E. J.: Interpretation of discordant U-Pb zircon ages; an evaluation, J. Metamorph. Geol., 15(1), 127-140, 1997.

Miller, E. L., Toro, J., Gehrels, G., Amato, J. M., Prokopiev, A., Tuchkova, M. I., Akinin, V. V., Dumitru, T. A., Moore, T. E., and Cecile, M. P.: New Insights into Arctic paleogeography and tectonics from $\mathrm{U}-\mathrm{Pb}$ detrital zircon geochronology, Tectonics, 25, TC3013, doi:10.1029/2005TC001830, 2006.

Miller, E. L., Soloviev, A., Kuzmichev, A., Gehrels, G., Toro, J. and Tuchkova, M.: Jura-Cretaceous foreland basin deposits of the Russian Arctic: Separated by birth of Makarov Basin?, Norw. J. Geol., 88, 227-250, 2008.

Miller, E. L. and Verzhbitsky, V. E: Structural studies near Pevek, Russia: Implications for formation of the East Siberian Shelf and Makarov Basin of the Arctic Ocean, in: Origins of Northeastern Russia: Paleomagnetism, Geology and Tectonics: A volume in honor of L. Parfenov, edited by: Stone, D. B., Miller, E. L., Toro, J., et al., Stephan Mueller Spec. Publ. Ser., this volume.

Muir, R. J., Ireland, T. R., Weaver, S. D., and Bradshaw, J.D.: Ion microprobe dating of Paleozoic granitoids: Devonian magmatism in New Zealand and correlations with Australia and Antarctica, Chem. Geol., 127(1-3), 191-210, 1996.

Natal'in, B. A., Amato, J. M., Toro, J., and Wright, J. E.: Paleozoic rocks of northern Chukotka Peninsula, Russian Far East; implications for the tectonics of the Arctic region, Tectonics, 18, 977-1003, 1999.

Nokleberg, W. J., Parfenov, L. M., Monger, J., et al.: Circum-North Pacific tectono-stratigraphic terrane map, US Geological Survey Open-File Report 94-714, 2 sheets, scale 1:5000 000, 433 pp., 1994.

Nokleberg, W. J., Parfenov, L. M., Monger, J. W. H., Norton, I. O., Khanchuk, A. I., Stone, D. B., Scholl, D. W., and Fujita, K.: Phanerozoic Tectonic Evolution of the Circum-North Pacific, US
Geological Survey Open-File Report 98-754, 126 pp., 1998.

Parfenov, L. M.: Continental margin and island-arcs of the Mesozoids, northeast Asia: Novosibersk, Nauka, 192 pp., 1984.

Parfenov, L. M.: Tectonics of the Verkhoyansk-Kolyma Mesozoids in the context of plate tectonics, Tectonophysics, 199, 319-342, 1991.

Parfenov, L. M.: Accretionary history of northeast Asia, in: Proceedings of the 1994 International Conference on Arctic Margins, edited by: Thurston, D. and Fujita, K., US Dep. Int., Min. Manag. Serv., Anchorage, 183-188, 1994.

Rowley, D. B. and Lottes, A. L.: Plate-kinematic reconstructions of the North Atlantic and Arctic: Late Jurassic to Present, Tectonophysics 155, 73-120, 1988.

Sadovskiy, A. I. and Gel'man, M. L.: The geological map of the USSR, Anyuisko-Chaunskaya series, Explanatory note, Sheet RXXVII, XXVIII, scale 1:200 000, 1970.

Seslavinskiy, K. B.: The south Anyui suture (western Chukotka), Dokl. Akad. Nauk SSSR+, 249, 1181-1185, 1979 (in Russian).

Sokolov, S. D., Bondarenko, G. Ye., Morozov, O. L., Shekhovtsov, V. A., Glotov, S. P., Ganelin, A. V., Kravchenko, B., and Berezhnoy, I. R.: South Anyui suture, Northeast Arctic Russia; facts and problems, in: Tectonic Evolution of the Bering Shelf-Chukchi Sea-Arctic Margin and Adjacent Landmasses, edited by: Miller, E. L., Grantz, A., and Klemperer, S. L., Geol. Soc. Am. Special Paper, 360, 209-224, 2002.

Sosunov, G. M. and Til'man, S. M. (Eds.): Geologic map of USSR: North-Eastern Geological Directorate, Anyuisko-Chaunskaya series, R-58-XXXV, XXXVI, 1 sheet, scale 1:200 000, 1960.

Tera, F. and Wasserburg, G. J.: U-Th-Pb systematics on lunar rocks and inferences about lunar evolution and the age of the Moon, in: Proceedings of 5th Lunar Sci. Conf. (Supp. 5, Geochim. Cosmochim. Acta), 2, 1571-99, 1974.

Tikhomirov, P. L., Akinin, V. V., Ispolatov, V. O., Alexander, P., Cherepanova, I. Yu., and Zagoskin, V. V.: The OkhotskChukotka Volcanic Belt: Age of its northern part according to new $\mathrm{Ar}-\mathrm{Ar}$ and $\mathrm{U}-\mathrm{Pb}$ geochronological data, Stratigr. Geo. Correl., 14(5), 524-537, 2006.

Tuchkova, M. I., Bondarenko, G. E., Buyakaite, M. I., Golovin, D. I., Galuskina, I. O., and Pokrovskaya, E. V.: Deformation of the Chukchi microcontinent: Structural, lithologic and geochronologic evidence, Geotectonics, 41(5), 403-421, 2007.

Tuchkova, M. I., Miller, E. L., Bondarenko, G. Y., Gehrels, G. E., Bujakaite, M. I., and Golovin, D. I.: Paleogeographical reconstruction on Triassic sedimentary basin of western Chukotka: Facial, petrographical and isotopic data, in: Sedimentary processes: Sedimentation, lithogenesis and ore generation, 1, 203-04, 2006 (in Russian).

Verts, L. A., Chamberlain, K. R., and Frost, C. D.: U-Pb sphene dating of metamorphism: the importance of sphene growth in the contact aureole of the Red Mountain pluton, Laramie Mountains, Wyoming, Contr. Min. Pet., 125, 186-199, 1996.

Unruh, J. R., Dumitru, T. A., and Sawyer, T. L.: Coupling of early Tertiary extension in the Great Valley forearc basin with blueschist exhumation in the underlying Franciscan accretionary wedge at Mt. Diablo, California, Geol. Soc. Am. Bull., 119, 1347-1367, 2007.

Williams, I. S.: U-Th-Pb geochronology by ion microprobe: not just ages but histories, Soc. Econ. Geol. Rev. Econ. Geol., 7, 1$35,1997$. 